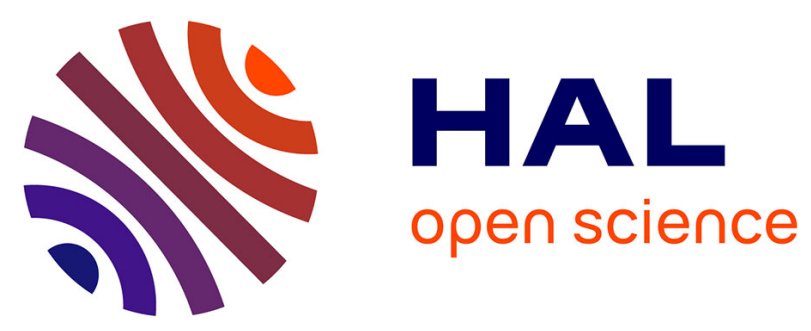

\title{
A LATIN-based model reduction approach for the simulation of cycling damage
}

Mainak Bhattacharyya, Amélie Fau, Udo Nackenhorst, David Néron, Pierre

Ladevèze

\section{- To cite this version:}

Mainak Bhattacharyya, Amélie Fau, Udo Nackenhorst, David Néron, Pierre Ladevèze. A LATINbased model reduction approach for the simulation of cycling damage. Computational Mechanics, 2018, 62 (4), pp.725-743. 10.1007/s00466-017-1523-z . hal-01832347

\section{HAL Id: hal-01832347 \\ https://hal.science/hal-01832347}

Submitted on 19 Aug 2019

HAL is a multi-disciplinary open access archive for the deposit and dissemination of scientific research documents, whether they are published or not. The documents may come from teaching and research institutions in France or abroad, or from public or private research centers.
L'archive ouverte pluridisciplinaire HAL, est destinée au dépôt et à la diffusion de documents scientifiques de niveau recherche, publiés ou non, émanant des établissements d'enseignement et de recherche français ou étrangers, des laboratoires publics ou privés. 


\title{
A LATIN-based model reduction approach for the simulation of cycling damage
}

\author{
Mainak Bhattacharyya ${ }^{1,2} \cdot$ Amelie Fau $^{1} \cdot$ Udo Nackenhorst ${ }^{1} \cdot$ David Néron $^{2} \cdot$ Pierre Ladevèze $^{2}$
}

\begin{abstract}
The objective of this article is to introduce a new method including model order reduction for the life prediction of structures subjected to cycling damage. Contrary to classical incremental schemes for damage computation, a non-incremental technique, the LATIN method, is used herein as a solution framework. This approach allows to introduce a PGD model reduction technique which leads to a drastic reduction of the computational cost. The proposed framework is exemplified for structures subjected to cyclic loading, where damage is considered to be isotropic and micro-defect closure effects are taken into account. A difficulty herein for the use of the LATIN method comes from the state laws which can not be transformed into linear relations through an internal variable transformation. A specific treatment of this issue is introduced in this work.
\end{abstract}

Keywords LATIN method · Proper Generalised Decomposition · Non-linear solid mechanics · Damage · Reduced order model

\section{Introduction}

In engineering problems, the failure of structures is often governed by the creation and growth of micro-voids and micro-cracks. These micro-level discontinuities can coalesce and grow into macro-cracks which may propagate through structures resulting in failure. They are quantified in continuum scale as a homogenised quantity described by an internal variable. Damage, in its mechanical sense, is the creation and growth of micro-voids or micro-cracks. The representation of damage as a scalar variable was first introduced by Kachanov [20]. Many works have been followed since then, notably by Lemaitre and Desmorat $[30,32]$. For ductile materials, isotropic damage can be physically interpreted as a volume density of micro-voids, and also as reduction in the stiffness of the material [32]. Mostly, the isotropic damage variable is defined between 0 and 1 [20,30,32,37], where 0 represents a virgin material and 1 represents complete failure. It is, however, also possible to introduce a damage variable

Mainak Bhattacharyya

mainak.bhattacharyya@ibnm.uni-hannover.de

1 IBNM, Leibniz Universität Hannover, Appelstraße 9a, 30167 Hannover, Germany

2 LMT, ENS Paris-Saclay, CNRS, Université Paris-Saclay, 61 avenue du Président Wilson, 94235 Cachan, France between 0 (undamaged) and $\infty$ (completely damaged) [5]. The phenomenon of low cycle fatigue (LCF) is governed by macro-plasticity (or visco-plasticity), where the dissipative energy for damage exists at the macro-scale [33]. For high cycle fatigue (HCF), the structure is macroscopically elastic, however plasticity and damage exist at the microscale. In the presence of cyclic loading, damage during tension is much higher than during compression because of the closure of the micro-voids and the material recovers its stiffness, partially or completely [32]. For solving this type of problems numerically, most of the developments in the field of continuum damage mechanics are based on a classical time incremental framework [32,33]. Numerical challenges rely on the accuracy of the required iterative algorithm as well as on its stability when damage increases [13] or on the efficient computation of the response under cyclic loading $[12,18,35,48]$.

For the last decades, model order reduction has been appealing for many mechanical problem to tackle large size problems, real-time computations or parametric studies $[8,15]$. For that purpose, the idea of model reduction is to seek the solution of a given problem in a reduced-order basis, whose dimension is much smaller than the size of the original high-dimensional model. This idea takes advantage of the redundancy of information that usually exists when describing the solution. Model reduction techniques based on the 
Proper Orthogonal Decomposition (POD) [4,7,8,22], involve a learning phase which consists in solving the full-order problem at some particular time instants and/or parameter values arbitrarily chosen. These solutions, called snapshots, are used to build reduced-order basis, by means of truncated POD, and a reduced-order model is then generated (e.g. by Galerkin projection) to solve the problem for the entire time and/or parameter domain. Since the 80's, the Reduced-Basis (RB) approach has been developed and consists of a greedy algorithm which selects the most relevant calculations to be performed on the parametric space in order to enrich the reduced-order basis $[17,34,39,40,44,45,49]$. Another appealing family of model reductions which has received a growing interest during the last decade is based on the Proper Generalised Decomposition (PGD). Roughly, PGD also consists in seeking the solution of a problem in a relevant reduced-order basis but this one is generated automatically and on-the-fly by a greedy algorithm, simultaneously with the successive approximations of the solution. The interested reader is referred to $[24,26]$ for structural non-linear problems and the works of Chinesta and co-authors to solve parameterised problems $[1,3,16]$. An extensive review of the literature on this method can be found in [10].

Computation of continuum damage problems may lead to solution bifurcation or to strain localisation. Because such behaviours are highly sensible to any modification of the model, computational strategies using model order reduction for damage are challenging and may be hazardous. It is generally recommended to control with circumspection, the accuracy of the solution provided by computations based on POD and to prefer some adaptive schemes as A Priori Hyper Reduction Method [47] or POD coupled with Newton-Krylov algorithms [21]. PGD also offers such a flexibility as used recently by [36], which introduced the coupling of PGD with a cohesive zone for delamination, or [14] for multi-scale computations for simulating problems with a rate-dependent damage model. These different authors have shown that model order reduction are promising to forecast damage evolution with limited computational cost.

In the current article, a different approach is proposed, based on the LATIN method [24] which looks for an approximation of the solution on the entire time-space domain at every iteration. Therefore, this method is said to be nonincremental in time although discretisation schemes in space and in time are used as usual. As it handles an approximation of the whole time-space domain at every iteration, this approach is very suitable to include model order reduction techniques even for non-linear computations. A drastic decrease of the computational cost compared to a classical approach would allow to tackle sophisticated cases such as cyclic loading for example.
The LATIN-PGD has been developed for solving plasticity and visco-plasticity problems, even with cycling loading. From the first works $[11,23,24]$, performances and robustness have improved thanks to [26-28,38,43], leading to a mature approach for classical (visco-)plastic problems with parameters. The aim of this paper is to extend the approach for (visco-)plastic problems with unilateral damage. A major difficulty comes from the state laws which can not be transformed into linear relations through an internal variable transformation in order to employ the usual LATIN-PGD framework. An extension of the algorithm is introduced for the treatment of this non-linearity. The powerful numerical framework offered by LATIN-PGD seems to be robust and efficient to also predict damage including intrinsic model order reduction.

The article is structured as follows. In Sect. 2, the general problem is introduced in time-space domain with corresponding admissibility conditions. Constitutive formulations for the state and internal variables are presented including damage evolutions. In Sect. 3 the innovative LATIN algorithm is presented, incorporating damage. In Sect. 4, the PGD technique is described. The stress and strain are mathematically separated in terms of a part that depends on the plastic strain and the other part that depends on damage. The former is represented in a separable form of space and time which is estimated using a hybrid technique. Finally, two numerical examples are detailed in Sect. 5 to analyse the performances of the new algorithm.

\section{The reference problem}

The reference problem is a quasi-static isothermal evolution of a structure defined over time-space domain $[0, T] \times \Omega$, assuming small perturbation. The structure is subjected to prescribed body forces $\underline{f}_{d}$, to traction forces $\underline{F}_{d}$ over a part $\partial_{2} \Omega$ of the boundary. The structure is also subjected to prescribed displacements $\underline{u}_{d}$ over the complementary part $\partial_{1} \Omega$ (Fig. 1). The state of the structure is defined by the set of field variables $\boldsymbol{s}=\left\{\dot{\boldsymbol{\varepsilon}}^{p}, \boldsymbol{\varepsilon}^{e}, \dot{\boldsymbol{X}}, \dot{D}, \boldsymbol{\sigma}, \boldsymbol{Z}, Y\right\}$, where

$-\boldsymbol{\varepsilon}^{p}$ is the inelastic part of the total strain $\boldsymbol{\varepsilon}$ corresponding to the displacement field $\underline{u}$, that satisfies the strain partition relation $\varepsilon=\varepsilon^{e}+\varepsilon^{p}$, with $\boldsymbol{\varepsilon}^{e}$ being the elastic strain. $\boldsymbol{X}$ represents the internal variables related to hardening. $D$ is the isotropic damage variable associated with damaged state of the material.

- $\boldsymbol{\sigma}$ is the Cauchy stress, $\boldsymbol{Z}$ is the set of variables conjugate to $X$, i.e. the thermodynamic forces associated to $X$. $Y$ represents the energy release rate associated with damage and is the conjugate variable of $D$. 


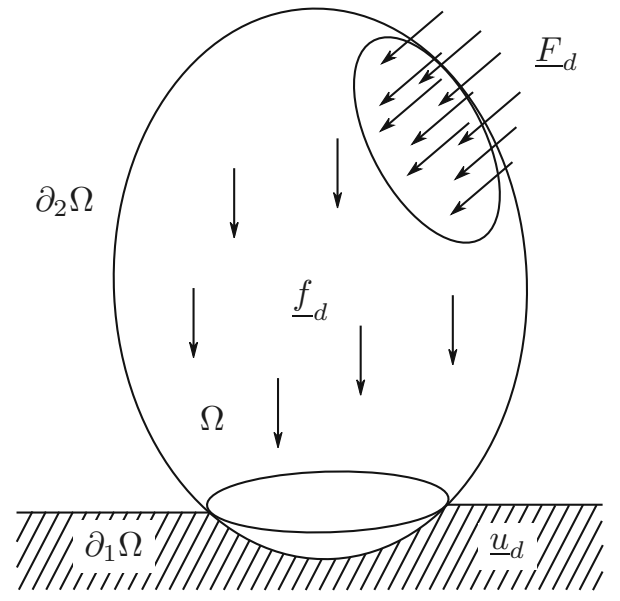

Fig. 1 Reference problem in domain $\Omega$

\subsection{Constitutive relations}

The formulation of constitutive relations involves the description of the state equations and the formulation of the evolution laws.

\subsubsection{State equations}

The equations of state are obtained from the Gibbs free energy function $\psi$ which can be decoupled into an elastic damage part $\psi^{e}$ and a plastic hardening part $\psi^{p}$,

$\rho \psi=\rho \psi^{e}(\boldsymbol{\sigma}, D)+\rho \psi^{p}(\boldsymbol{X})$,

where $\rho$ is the mass density. The difference in the elastic state equation during tension and compression is due to the fact that during compression the material regains some stiffness, as some of the micro-defects are closed, thereby increasing the effective area. This can be represented by the effective modulus of elasticity during tension $\tilde{E}^{+}$, which is given by $\tilde{E}^{+}=E(1-D)$, where $E$ is the true modulus of elasticity, and by the effective elastic modulus during compression $\tilde{E}^{-}$given by $\tilde{E}^{-}=E(1-h D)$. The closure parameter $h$ has values between 0 (complete stiffness recovery) and 1 (no stiffness recovery). This phenomenon is described by an elastic free energy function given as

$$
\begin{aligned}
\rho \psi^{e}= & \frac{1+v}{2 E}\left[\frac{\langle\sigma\rangle_{i j}^{+}\langle\sigma\rangle_{i j}^{+}}{1-D}+\frac{\langle\sigma\rangle_{i j}^{-}\langle\sigma\rangle_{i j}^{-}}{1-h D}\right] \\
& -\frac{v}{2 E}\left[\frac{\left\langle\sigma_{k k}\right\rangle^{2}}{1-D}+\frac{\left\langle-\sigma_{k k}\right\rangle^{2}}{1-h D}\right],
\end{aligned}
$$

where $v$ is the Poisson ratio. Hardening in classical sense is classified into isotropic and kinematic hardening. Isotropic hardening is the phenomenon where there is an increase in the radius of the yield surface within the $\Pi$ plane and the centre of the yield surface remains constant. Kinematic hardening is where the yield surface translates in the $\Pi$ plane without any change in radius. For metals, pure isotropic hardening can not describe Bauschinger effect but pure kinematic hardening can. For practical cases, however a combination of the two (mixed hardening) is used [31]. For mixed hardening case the plastic free energy function can be written as

$\rho \psi^{p}(\boldsymbol{X})=\rho \psi^{p}(\boldsymbol{\alpha}, r)=\frac{1}{2}\left[\alpha_{i j} C \alpha_{i j}\right]+g(r)$,

where $\alpha$ is internal variable corresponding to kinematic hardening, $r$ is the internal variable for isotropic hardening. $\boldsymbol{\beta}$ and $R$ are conjugate to $\alpha$ and $r$ respectively. $C$ is a material parameter for kinematic hardening and $g(r)$ is a function describing the isotropic hardening. These definitions give the state equations as

$$
\begin{aligned}
\varepsilon_{i j}^{e}=\rho \frac{\partial \psi^{e}}{\partial \sigma_{i j}}= & \frac{1+v}{E}\left[\frac{\langle\sigma\rangle_{i j}^{+}}{1-D}+\frac{\langle\sigma\rangle_{i j}^{-}}{1-h D}\right] \\
& -\frac{v}{E}\left[\frac{\left\langle\sigma_{k k}\right\rangle}{1-D}+\frac{\left\langle-\sigma_{k k}\right\rangle}{1-h D}\right] \delta_{i j}, \\
\beta_{i j}=\rho \frac{\partial \psi^{p}}{\partial \alpha_{i j}}= & C \alpha_{i j}, \\
R=\rho \frac{\partial \psi^{p}}{\partial r}= & g^{\prime}(r), \\
Y=\rho \frac{\partial \psi^{e}}{\partial D}= & \frac{1+v}{2 E}\left[\frac{\langle\sigma\rangle_{i j}^{+}\langle\sigma\rangle_{i j}^{+}}{(1-D)^{2}}+h \frac{\langle\sigma\rangle_{i j}^{-}\langle\sigma\rangle_{i j}^{-}}{(1-h D)^{2}}\right] \\
& -\frac{v}{2 E}\left[\frac{\left\langle\sigma_{k k}\right\rangle^{2}}{(1-D)^{2}}+h \frac{\left\langle-\sigma_{k k}\right\rangle^{2}}{(1-h D)^{2}}\right] .
\end{aligned}
$$

It has to be noticed that the linear relationship $\varepsilon^{e}=\mathbf{C}^{-1} \sigma$ between stress and elastic strain field, with $\mathbf{C}$ being the Hooke tensor, is modified in Eq. (4a) to include unilateral damage. An exponential isotropic rule is generally considered as,

$g^{\prime}(r)=R_{\infty}[1-\exp (-\gamma r)]$

with $R_{\infty}$ and $\gamma$ being material parameters describing isotropic hardening.

\subsubsection{Evolution laws}

The starting point of the formulation of the evolution equations is the second law of thermodynamics, written as Clausius-Duhem inequality,

$\sigma_{i j} \dot{\varepsilon}_{i j}^{p}-R \dot{r}-\beta_{i j} \dot{\alpha}_{i j}+Y \dot{D} \geqslant 0$. 
The evolution equations for the internal variables are obtained from a dissipation potential $\phi$, which is a convex function of the associated variables to ensure that Eq. (6) is satisfied. The dissipation potential can be decoupled as

$\phi=\phi^{p}+\phi^{d}$

where $\phi^{p}$ represents the dissipation due to plasticity and $\phi^{d}$ represents the dissipation due to damage. For visco-plasticity $\phi^{p}$ can be defined according to Norton's law as

$\phi^{p}=\frac{k}{n+1}\left\langle f^{p}\right\rangle_{+}^{n+1}$,

where $k$ and $n$ are material dependent viscous coefficient and exponent respectively and $f^{p}$ is the yield function. Considering $\tau_{i j}=\frac{\sigma_{i j}^{D}}{1-D}-\beta_{i j}$, with $\sigma_{i j}^{D}$ being the deviatoric part of the stress tensor, the yield function for $J_{2}$ plasticity in a Marquis-Chaboche model is defined as,

$f^{p}=\sqrt{\frac{3}{2} \tau_{i j} \tau_{i j}}+\frac{a}{2 C}\left[\beta_{i j} \beta_{i j}\right]-R-\sigma_{y}$.

The second term is specific to Marquis-Chaboche model and indicates material softening, where $a$ is a material parameter and $\sigma_{y}$ is the yield stress. The evolution equations can then be written as

$$
\begin{aligned}
\dot{\varepsilon}_{i j}^{p} & =\frac{\partial \phi^{p}}{\partial \sigma_{i j}}=k\left\langle f^{p}\right\rangle_{+}^{n}\left[\frac{3}{2} \frac{\tau_{i j}}{\sqrt{\frac{3}{2} \tau_{i j} \tau_{i j}}}\right] \frac{1}{1-D}, \\
\dot{\alpha}_{i j} & =-\frac{\partial \phi^{p}}{\partial \beta_{i j}}=-k\left\langle f^{p}\right\rangle_{+}^{n}\left[-\frac{3}{2} \frac{\tau_{i j}}{\sqrt{\frac{3}{2} \tau_{i j} \tau_{i j}}}+\frac{a}{C} \beta_{i j}\right], \\
\dot{r} & =-\frac{\partial \phi^{p}}{\partial R}=-k\left\langle f^{p}\right\rangle_{+}^{n}(-1) .
\end{aligned}
$$

Similar to plasticity, the damage potential $\phi^{d}$ can be defined as

$\phi^{d}=\frac{k_{d}}{n_{d}+1}\left\langle f^{d}\right\rangle_{+}^{n_{d}+1}$,

with $k_{d}$ and $n_{d}$ being the damage coefficient and damage exponent respectively. $f_{d}$ is the damage yield function given by,

$f^{d}=Y-Y_{0}$

where $Y_{0}$ describes the damage threshold. The evolution equation for damage is then given by

$\dot{D}=\frac{\partial \phi^{d}}{\partial Y}=k_{D}\left\langle f^{d}\right\rangle_{+}^{n_{d}}$.
A similar damage evolution equation had been proposed in [5], where an unbounded damage variable defined between 0 and $\infty$ was used. The damage evolution law (13) is also thermodynamically consistent for bounded damage variable defined between 0 and 1 . Due to the presence of macroplasticity, it can be considered that damage occurs only during plastic deformation and there is no evolution of damage in the elastic regime, which defines the threshold $Y_{0}$ as

$Y_{0}=\frac{\sigma_{y}^{2}}{2 E}$

\subsection{Admissibility conditions}

For the admissibility conditions of the reference problem, the following spaces and the corresponding vector spaces (denoted with subscript 0 ) are defined:

- The space $\mathcal{U}$ of the kinematic admissibility fields $\underline{u}$ such that

$\underline{u}_{\mid t=0}=\underline{u}_{0} \quad$ and $\quad \underline{u}=\underline{u}_{d}$ on $\partial_{1} \Omega$.

- The space $\mathcal{S}$ of the statically admissible fields $\sigma$ such that,

$$
\begin{gathered}
\int_{[0, T] \times \Omega} \sigma: \boldsymbol{\varepsilon}\left(\underline{u}^{*}\right) \mathrm{d} \Omega \mathrm{d} t=\int_{[0, T] \times \Omega} \underline{f} \underline{f}_{d} \cdot \underline{u}^{*} \mathrm{~d} \Omega \mathrm{d} t \\
+\int_{[0, T] \times \partial_{2} \Omega} \underline{F}_{d} \cdot \underline{u}^{*} \mathrm{~d} S \mathrm{~d} t, \quad \forall \underline{u}^{*} \in \mathcal{U}_{0} .
\end{gathered}
$$

- The space $\mathcal{E}$ of the kinematically admissible fields $\boldsymbol{\varepsilon}$ such that $\exists \underline{u} \in \mathcal{U}, \boldsymbol{\varepsilon}=\nabla_{\text {sym }} \underline{u}$, which in weak form can be written as,

$$
\begin{aligned}
& \int_{[0, T] \times \Omega} \sigma^{*}: \boldsymbol{\varepsilon} \mathrm{d} \Omega \mathrm{d} t \\
& \quad=\int_{[0, T] \times \partial_{1} \Omega} \sigma^{*} \underline{n} \cdot \underline{u}_{d} \mathrm{~d} S \mathrm{~d} t, \quad \forall \boldsymbol{\sigma}^{*} \in \mathcal{S}_{0} .
\end{aligned}
$$

\subsection{Partial normal formulation}

To use efficiently the LATIN method as a solver, the state equations should be described by linear operators. Hence it is necessary to introduce at this point a "normal" formulation of the constitutive relations [11,24]. For that purpose, the state law (4c) is transformed into linear relations by a change of variable

$\bar{R}=R_{\infty} \bar{r}$

where, $\bar{R}$ and $\bar{r}$ are the new isotropic variables, with 


$$
\begin{aligned}
\bar{r} & =\frac{2}{\gamma^{1 / 2}}\left[1-\exp \left(-\frac{\gamma r}{2}\right)\right], \\
R & =R_{\infty}\left(\frac{\bar{R}}{R_{\infty}} \frac{\gamma^{1 / 2}}{2}\right)\left(2-\frac{\bar{R}}{R_{\infty}} \frac{\gamma^{1 / 2}}{2}\right) .
\end{aligned}
$$

Using these two new variables, the yield function becomes,

$$
\begin{aligned}
f^{p}= & \sqrt{\frac{3}{2} \tau_{i j} \tau_{i j}}+\frac{a}{2 C}\left[\beta_{i j} \beta_{i j}\right] \\
& -R_{\infty}\left(\frac{\bar{R}}{R_{\infty}} \frac{\gamma^{1 / 2}}{2}\right)\left(2-\frac{\bar{R}}{R_{\infty}} \frac{\gamma^{1 / 2}}{2}\right)-\sigma_{y},
\end{aligned}
$$

and the evolution Eq. (10c) can be written as,

$\dot{\bar{r}}=-k\left\langle f^{p}\right\rangle_{+}^{n}\left(\frac{\bar{R}}{R_{\infty}} \frac{\gamma}{2}-\gamma^{1 / 2}\right)$.

However, it has to be noted that the elasticity law (4a) cannot be transformed into a linear relation, due to the damage term, and it will require a specific treatment.

\section{The LATIN framework}

Solving the reference problem has three difficulties; first being satisfying the global equilibrium of the structure, the second being taking into account the non-linear elastic law, and finally to solve the non-linear evolution equations. The LATIN method is a non-incremental solver in time that tackles these sets of equations iteratively on the whole time-space domain. The algorithm is initialised by the solution of the reference problem considering the loading is elastic and then plastic and damage corrections are added iteratively. For that purpose, two manifolds are introduced, based on a subdivision of the set of equations that must be solved:

- the first is the space $\boldsymbol{A}$ which belongs to the manifold of the admissibility conditions $(15,16,17)$, the linear state laws $(4 b, 18)$, and also the non-linear state law for damage (4d), that although being non-linear, can be postprocessed from the stress tensor and the damage variable at the end of each iteration;

- the second is the space $\boldsymbol{\Gamma}$ which belongs to the manifold of the evolution equations $(10 \mathrm{a}, 10 \mathrm{~b}, 13,21)$ and the elastic state law (4a) which was not linearisable due to damage.

The exact solution is then given by

$$
\boldsymbol{s}_{e x} \in \boldsymbol{A} \cap \boldsymbol{\Gamma} .
$$

To find $\boldsymbol{s}_{e x}$, an iterative algorithm is used, which consists, at each iteration, of a non-linear local stage and a linear global stage, seeking alternatively for an approximation of the solution field $\boldsymbol{s}$ over $\boldsymbol{\Gamma}$ and $\boldsymbol{A}$. The relation between the two spaces are governed by linear operators called search directions. The iterative algorithm can be represented as follows:

$$
\begin{aligned}
& \boldsymbol{s}_{0} \in \boldsymbol{A} \longrightarrow \hat{\boldsymbol{s}}_{1 / 2} \in \boldsymbol{\Gamma} \cdots \longrightarrow \hat{\boldsymbol{s}}_{i+1 / 2} \in \boldsymbol{\Gamma} \\
& \longrightarrow \boldsymbol{s}_{i+1} \in \boldsymbol{A} \cdots \longrightarrow \boldsymbol{s}_{\text {ex }} .
\end{aligned}
$$

An overview of the extended method including unilateral damage is schematised in Fig. 2.

\subsection{Initialisation}

The beginning of the algorithm starts with an elastic initialisation. The loading is considered to be elastic and a solution set $s_{0} \in \boldsymbol{A}$ is calculated. Due to the elastic assumption, all the internal variables $\boldsymbol{X}, \boldsymbol{Z}, D$ and $\boldsymbol{\varepsilon}^{p}$ are set equal to zero. All the boundary conditions are taken into account in the initialisation stage. At each subsequent iteration, correction to the elastic solution is computed.

\subsection{Local stage}

The objective of the local stage is to solve the evolution equations for internal variables that are local in space and non-linear. The elastic state law, being non-linear is also treated in this stage. The solution field $s$ is separated into two parts $\boldsymbol{s}^{p}=\left\{\dot{\boldsymbol{\varepsilon}}^{p}, \boldsymbol{\varepsilon}^{e}, \dot{\boldsymbol{X}}, \boldsymbol{\sigma}, \boldsymbol{Z}\right\}$ and $\boldsymbol{s}^{d}=\{\dot{D}, Y\}$. Both solution fields are solved using linear operators having different properties. Knowing a solution set $\boldsymbol{s}_{i} \in \boldsymbol{A}$, the objective is to find $\hat{\boldsymbol{s}}_{i+1 / 2} \in \boldsymbol{\Gamma}$ such that the local search directions (directions of ascent) are satisfied

$$
\begin{aligned}
& {\left[\begin{array}{c}
\hat{\dot{\boldsymbol{\varepsilon}}}_{i+1 / 2}^{p}-\dot{\boldsymbol{\varepsilon}}_{i}^{p} \\
-\left(\hat{\boldsymbol{X}}_{i+1 / 2}-\dot{\boldsymbol{X}}_{i}\right) \\
\hat{\boldsymbol{\varepsilon}}_{i+1 / 2}^{e}-\boldsymbol{\varepsilon}_{i}^{e}
\end{array}\right]+\mathbf{B}^{+}\left[\begin{array}{c}
\hat{\boldsymbol{\sigma}}_{i+1 / 2}-\boldsymbol{\sigma}_{i} \\
\hat{\boldsymbol{Z}}_{i+1 / 2}-\boldsymbol{Z}_{i} \\
\hat{\boldsymbol{\sigma}}_{i+1 / 2}-\boldsymbol{\sigma}_{i}
\end{array}\right]=0,} \\
& {\left[\hat{\dot{D}}_{i+1 / 2}-\dot{D}_{i}\right]+\mathbf{b}^{+}\left[\hat{Y}_{i+1 / 2}-Y_{i}\right]=0 .}
\end{aligned}
$$

Here, $\mathbf{B}^{+}$and $\mathbf{b}^{+}$are the directions of ascent for solution sets $\boldsymbol{s}^{p}$ and $\boldsymbol{s}^{d}$ respectively. Following [24], the choice of these search directions is given by

$$
\left(\mathbf{B}^{+}\right)^{-1}=0 \quad, \quad\left(\mathbf{b}^{+}\right)^{-1}=0
$$

The solution of the search direction equations (24) along with the evolution Eqs. (10) and (13) and the non-linear elastic law (4a) gives easily the complete set of solution $\hat{\boldsymbol{s}}_{i+1 / 2}$. Knowing the solution at the local stage $\hat{\boldsymbol{s}}_{i+1 / 2}$, the solution set $s_{i+1}$ will be obtained in the global stage. 


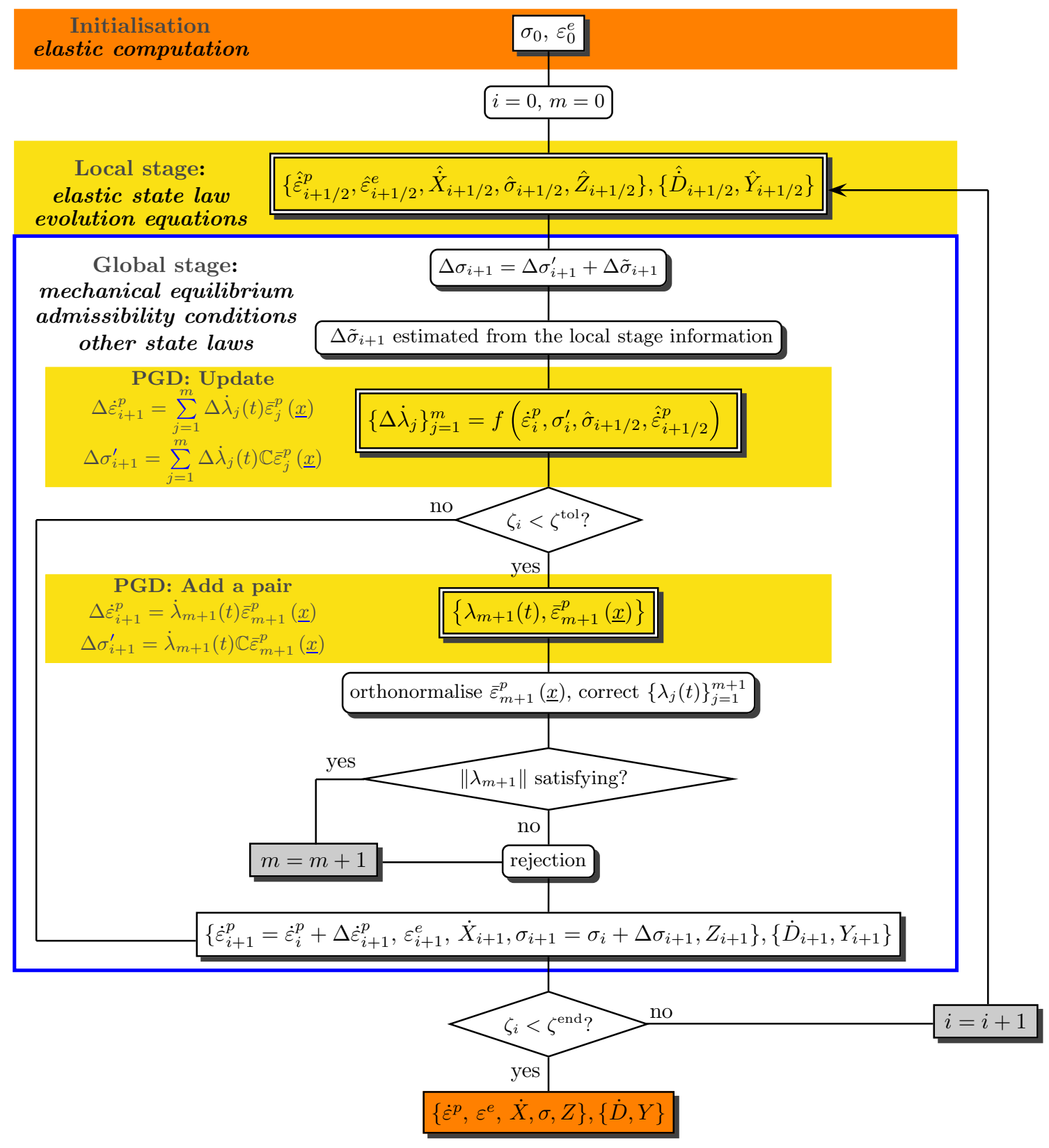

Fig. 2 Scheme of the innovative LATIN algorithm including unilateral damage ( $i$ : iteration of LATIN method, $m$ : number of PGD pairs involved in the separable form)

\subsection{Global stage}

In the global stage, apart from the state laws and admissibility conditions, the solution set $\boldsymbol{s}_{i+1} \in \boldsymbol{A}$ must also satisfy the descent search directions, i.e.

$$
\left[\begin{array}{c}
\dot{\boldsymbol{\varepsilon}}_{i+1}^{p}-\hat{\dot{\boldsymbol{\varepsilon}}}_{i+1 / 2}^{p} \\
-\left(\dot{\boldsymbol{X}}_{i+1}-\hat{\dot{\boldsymbol{X}}}_{i+1 / 2}\right) \\
\varepsilon_{i+1}^{e}-\hat{\varepsilon}_{i+1 / 2}^{e}
\end{array}\right]-\mathbf{B}^{-}\left[\begin{array}{c}
\sigma_{i+1}-\hat{\boldsymbol{\sigma}}_{i+1 / 2} \\
\boldsymbol{Z}_{i+1}-\hat{\boldsymbol{Z}}_{i+1 / 2} \\
\boldsymbol{\sigma}_{i+1}-\hat{\boldsymbol{\sigma}}_{i+1 / 2}
\end{array}\right]=0,
$$

$\left[\dot{D}_{i+1}-\hat{\dot{D}}_{i+1 / 2}\right]-\mathbf{b}^{-}\left[Y_{i}-\hat{Y}_{i+1 / 2}\right]=0$, 
where $\mathbf{B}^{-}=\left[\begin{array}{cc}\mathbf{H}^{-} & 0 \\ 0 & \mathbf{C}^{-1}\end{array}\right]$. The choice of the operator $\mathbf{H}^{-}$ is such that it belongs to the tangent space associated with the solution set $\hat{\boldsymbol{s}}_{i+1 / 2}$ in the manifold $\boldsymbol{\Gamma}$. It is defined as the second derivative of the visco-plastic dissipation potential $\phi^{p}$. For mixed hardening, this is of the form

$\mathbf{H}^{-}=\left[\begin{array}{ccc}\mathbf{H}_{\sigma} & \mathbf{H}_{\sigma \beta} & \mathbf{H}_{\sigma R} \\ \mathbf{H}_{\beta \sigma} & \mathbf{H}_{\beta} & \mathbf{H}_{\beta R} \\ \mathbf{H}_{R \sigma} & \mathbf{H}_{R \beta} & \mathbf{H}_{R}\end{array}\right]$.

For the sake of simplicity, only an approximation of the tangent is computed and the off-diagonal terms are considered to be zero, which results to

$\mathbf{H}^{-}=\left[\begin{array}{ccc}\mathbf{H}_{\sigma} & 0 & 0 \\ 0 & \mathbf{H}_{\beta} & 0 \\ 0 & 0 & \mathbf{H}_{R}\end{array}\right]=\left[\begin{array}{cc}\mathbf{H}_{\sigma} & 0 \\ 0 & \mathbf{H}_{Z}\end{array}\right]$,

where,

$\mathbf{H}_{Z}=\left[\begin{array}{cc}\mathbf{H}_{\beta} & 0 \\ 0 & \mathbf{H}_{R}\end{array}\right]$.

At each space-time point, the operator $\mathbf{H}^{-}$obtained from the viscoplastic potential is a symmetric matrix, positive but semi-definite so its inverse is not always defined. Hence it is necessary to regularise it by

$\mathbf{H}^{-}=\mathbf{H}^{-}+c \mathbf{M}^{-1}$

where $\zeta$ is the regularisation coefficient taken herein to be 0.15 , and

$\mathbf{M}=\left[\begin{array}{ccc}\mathbf{C} & 0 & 0 \\ 0 & C & 0 \\ 0 & 0 & R_{\infty}\end{array}\right]$.

The search direction operator for damage $\mathbf{b}^{-}$, is taken to be zero, that is the damage variable does not change in the linear stage.

The new difficulty, compared to former works with the LATIN method, is that the elastic state law (4a) is non-linear due to the presence of damage, leading to solve a non-linear problem at the global stage. This point is particularly tricky as it prevents the introduction of a model reduction strategy at this stage. The idea proposed herein is to transform this nonlinear problem into separate linear equations by decomposing stress and total strain into two parts depending on plastic deformation and damage respectively.
The quantities of interest at this point $\sigma_{i+1}, \boldsymbol{\varepsilon}_{i+1}^{e}$ and $\dot{\boldsymbol{\varepsilon}}_{i+1}^{p}$ are represented in a corrective form at iteration $i+1$ as

$$
\begin{aligned}
\Delta \boldsymbol{\sigma}_{i+1} & =\sigma_{i+1}-\sigma_{i}, \Delta \boldsymbol{\varepsilon}_{i+1}^{e}=\boldsymbol{\varepsilon}_{i+1}^{e}-\boldsymbol{\varepsilon}_{i}^{e} \text { and } \Delta \dot{\boldsymbol{\varepsilon}}_{i+1}^{p} \\
& =\dot{\boldsymbol{\varepsilon}}_{i+1}^{p}-\dot{\boldsymbol{\varepsilon}}_{i}^{p}
\end{aligned}
$$

The stress correction in the global stage at iteration $i+1$ is separated into a part $\Delta \sigma_{i+1}^{\prime}$ that depends on the plastic deformation and another part $\Delta \tilde{\boldsymbol{\sigma}}_{i+1}$ that is obtained from the non-linear state law,

$\Delta \boldsymbol{\sigma}_{i+1}=\Delta \boldsymbol{\sigma}_{i+1}^{\prime}+\Delta \tilde{\boldsymbol{\sigma}}_{i+1}$

Similarly the total strain correction $\Delta \varepsilon_{i+1}=\Delta \varepsilon_{i+1}^{e}+\Delta \varepsilon_{i+1}^{p}$ at iteration $i+1$ is also separated into a part $\Delta \boldsymbol{\varepsilon}_{i+1}^{\prime}$ that depends on plasticity and a part $\Delta \tilde{\boldsymbol{\varepsilon}}_{i+1}$ that depends on damage,

$\Delta \boldsymbol{\varepsilon}_{i+1}=\Delta \boldsymbol{\varepsilon}_{i+1}^{\prime}+\Delta \tilde{\boldsymbol{\varepsilon}}_{i+1}$.

Now, the corrective terms given by Eq. (32) help to rewrite the search direction equation $\mathbf{C}^{-1}$ as

$\Delta \boldsymbol{\sigma}_{i+1}=\mathbf{C} \Delta \boldsymbol{\varepsilon}_{i+1}^{e}-\Delta \mathbf{R}_{i+1}$,

where $\Delta \mathbf{R}_{i+1}$ represents a residual stress term at iteration $i+1$ and is given by

$\Delta \mathbf{R}_{i+1}=\left(\sigma_{i}-\hat{\boldsymbol{\sigma}}_{i+1 / 2}\right)-\mathbf{C}\left(\boldsymbol{\varepsilon}_{i}^{e}-\hat{\boldsymbol{\varepsilon}}_{i+1 / 2}^{e}\right)$.

From the previous separations along with the additive strain decomposition relation, it can be established that

$$
\begin{aligned}
& \Delta \boldsymbol{\sigma}_{i+1}^{\prime}+\Delta \tilde{\boldsymbol{\sigma}}_{i+1} \\
& \quad=\mathbf{C}\left(\Delta \boldsymbol{\varepsilon}_{i+1}^{\prime}-\Delta \boldsymbol{\varepsilon}_{i+1}^{p}\right)+\mathbf{C}\left(\Delta \tilde{\boldsymbol{\varepsilon}}_{i+1}-\Delta \boldsymbol{\varepsilon}_{i+1}^{R}\right),
\end{aligned}
$$

where $\Delta \boldsymbol{\varepsilon}_{i+1}^{R}$ can be interpreted as a residual strain obtained from non-linear state law at iteration $i+1$ and is given by

$\Delta \boldsymbol{\varepsilon}_{i+1}^{R}=\mathbf{C}^{-1} \Delta \mathbf{R}_{i+1}$.

On the other hand, if only the plastic part is considered, the search direction defined by Eq. (26a) is written as

$\Delta \dot{\boldsymbol{\varepsilon}}_{i+1}^{p}-\mathbf{H}_{\sigma} \Delta \boldsymbol{\sigma}_{i+1}+\overline{\boldsymbol{\Delta}}_{i+1}=0$,

with

$\overline{\boldsymbol{\Delta}}_{i+1}=\mathbf{H}_{\sigma}\left(\hat{\boldsymbol{\sigma}}_{i+1 / 2}-\boldsymbol{\sigma}_{i}\right)-\left(\hat{\dot{\boldsymbol{\varepsilon}}}_{i+1 / 2}^{p}-\dot{\boldsymbol{\varepsilon}}_{i}^{p}\right)$. 
Due to the separation of the stress tensor, only the part depending on plasticity is included in Eq. (39), which can thereby be re-written as

$\Delta \dot{\boldsymbol{\varepsilon}}_{i+1}^{p}-\mathbf{H}_{\sigma} \Delta \boldsymbol{\sigma}_{i+1}^{\prime}+\overline{\boldsymbol{\Delta}}_{i+1}=0$.

The objective of the global stage is then to calculate $\left(\Delta \dot{\boldsymbol{\varepsilon}}_{i+1}^{p}, \Delta \boldsymbol{\sigma}_{i+1}, \Delta \boldsymbol{\varepsilon}_{i+1}^{e}\right)$ by solving the weak form of the equilibrium equation.

\section{PGD formulation of the global stage}

The Proper Generalised Decomposition (PGD) is based on the idea that any quantity of interest which is dependent on several independent variables can be approximated as a sum of products of one-variable functions [9,24]. This decomposition includes thus an error due to the assumption of a separable form. For instance a field $v(\underline{x}, t)$ dependent on space and time variables is approximated as

$v(\underline{x}, t) \approx \sum_{i=1}^{n} v_{i}^{x}(\underline{x}) v_{i}^{t}(t)$

The usage of PGD in the LATIN framework lies in the fact that the quantities of interest are defined by Eq. (32) at every successive iteration on the whole time-space domain and are estimated from a linear problem, allowing easily to estimate them as separable forms. Although, this type of representation is general and can be applied to any given field [24], it is only efficient to solve a problem if the solution is separable. If is not the case, for example in the case of moving loads, some adaptations can be proposed to make this type of approximation efficient [2].

The boundary conditions have been taken into account in the elastic initialisation, so here the solution set searched in terms of corrections has to be kinematically admissible to zero, i.e. belonging to $\boldsymbol{A}_{0}$. The static admissibility condition (16) can be written as

$\int_{[0, T] \times \Omega} \Delta \sigma_{i+1}^{\prime}: \varepsilon\left(\underline{u}^{*}\right) \mathrm{d} \Omega \mathrm{d} t=0, \quad \forall \underline{u}^{*} \in \mathcal{U}_{0}$,

with

$\Delta \boldsymbol{\sigma}_{i+1}^{\prime}=\mathbf{C}\left(\Delta \boldsymbol{\varepsilon}_{i+1}^{\prime}-\Delta \boldsymbol{\varepsilon}_{i+1}^{p}\right)$

Also the part that is related to damage can be written in a similar weak form as

$\int_{[0, T] \times \Omega} \Delta \tilde{\boldsymbol{\sigma}}_{i+1}: \boldsymbol{\varepsilon}\left(\underline{u}^{*}\right) \mathrm{d} \Omega \mathrm{d} t=0, \quad \forall \underline{u}^{*} \in \mathcal{U}_{0}$, with

$\Delta \tilde{\boldsymbol{\sigma}}_{i+1}=\mathbf{C}\left(\Delta \tilde{\boldsymbol{\varepsilon}}_{i+1}-\Delta \boldsymbol{\varepsilon}_{i+1}^{R}\right)$

\subsection{Separable representation of the quantities of interest}

The quantities of interest represented in corrective forms are currently $\Delta \boldsymbol{\sigma}_{i+1}^{\prime}, \Delta \tilde{\boldsymbol{\sigma}}_{i+1}, \Delta \dot{\boldsymbol{\varepsilon}}_{i+1}^{p}, \Delta \boldsymbol{\varepsilon}_{i+1}^{e}$. The plastic strain rate is written as

$\Delta \boldsymbol{\varepsilon}_{i+1}^{p}=\lambda^{p}(t) \overline{\boldsymbol{\varepsilon}}^{p}(\underline{x}) \Rightarrow \Delta \dot{\boldsymbol{\varepsilon}}_{i+1}^{p}=\dot{\lambda}^{p}(t) \overline{\boldsymbol{\varepsilon}}^{p}(\underline{x})$.

The total strain that depends on plastic deformation and the corresponding displacement field can also be separated as

$\Delta \underline{u}_{i+1}=\lambda^{u}(t) \underline{\bar{u}}(\underline{x}) \Rightarrow \Delta \boldsymbol{\varepsilon}_{i+1}^{\prime}=\lambda^{u}(t) \bar{\varepsilon}(\underline{x})$.

For the following development, the indices are dropped for simplicity and used if necessary. Introducing the PGD approximation, the test field in Eq. (43) becomes $\underline{u}^{*}=$ $\lambda^{u *} \underline{\bar{u}}+\lambda^{u} \underline{\bar{u}}^{*}$ with $\underline{\bar{u}}^{*} \in \mathcal{U}_{0}$ and $\lambda^{u *}$ does not have any condition. The space-time problem then can be separated into a space problem and a time problem. The time problem consists in finding $\lambda^{u}$ such that $\forall \lambda^{u *}$,

$$
\begin{aligned}
\int_{[0, T]} \lambda^{u} \lambda^{u *} \mathrm{~d} t \int_{\Omega} \mathbf{C} \overline{\boldsymbol{\varepsilon}}: \boldsymbol{\varepsilon}(\underline{\bar{u}}) \mathrm{d} \Omega \\
\quad=\int_{[0, T]} \lambda^{p} \lambda^{u *} \mathrm{~d} t \int_{\Omega} \mathbf{C} \overline{\boldsymbol{\varepsilon}}^{p}: \boldsymbol{\varepsilon}(\underline{\bar{u}}) \mathrm{d} \Omega .
\end{aligned}
$$

This gives that $\lambda^{u}$ and $\lambda^{p}$ are proportional and the simplest choice is to assume $\lambda^{u}=\lambda^{p}=\lambda$. With this choice of the time function, the space problem simply consists in seeking $\underline{\bar{u}} \in \mathcal{U}_{0}$ such that

$$
\int_{\Omega} \mathbf{C} \overline{\boldsymbol{\varepsilon}}: \boldsymbol{\varepsilon}\left(\underline{\bar{u}}^{*}\right) \mathrm{d} \Omega=\int_{\Omega} \mathbf{C} \overline{\boldsymbol{\varepsilon}}^{p}: \boldsymbol{\varepsilon}\left(\underline{\bar{u}}^{*}\right) \mathrm{d} \Omega, \quad \forall \underline{\bar{u}}^{*} \in \mathcal{U}_{0} .
$$

This allows to define an operator $\mathbb{E}[43]$ such that

$\bar{\varepsilon}=\mathbb{E} \overline{\boldsymbol{\varepsilon}}^{p}$

Equation (44) can then be written as

$\Delta \boldsymbol{\sigma}^{\prime}=\lambda \mathbf{C}(\mathbb{E}-\mathbb{I}) \overline{\boldsymbol{\varepsilon}}^{p}=\lambda \mathbb{C} \overline{\boldsymbol{\varepsilon}}^{p}$,

with $\mathbb{C}=\mathbf{C}(\mathbb{E}-\mathbb{I})$ and $\mathbb{I}$ being the identity matrix. Finally including PGD in the global stage, at LATIN iteration $i+1$, 
the description of the quantities of interest depending on the plastic strain becomes

$\Delta \dot{\boldsymbol{\varepsilon}}_{i+1}^{p}=\dot{\lambda}(t) \overline{\boldsymbol{\varepsilon}}^{p}(\underline{x})$,

$\Delta \boldsymbol{\sigma}_{i+1}^{\prime}=\lambda(t) \mathbb{C} \overline{\boldsymbol{\varepsilon}}^{p}(\underline{x})$.

The quantities that depend on damage, namely $\Delta \tilde{\boldsymbol{\sigma}}_{i+1}$ and $\Delta \tilde{\boldsymbol{\varepsilon}}_{i+1}$, are obtained from the weak form of Eq. (45) along with Eq. (46) giving

$\int_{[0, T] \times \Omega} \mathbf{C}\left(\Delta \tilde{\boldsymbol{\varepsilon}}_{i+1}-\Delta \boldsymbol{\varepsilon}_{i+1}^{R}\right): \tilde{\boldsymbol{\varepsilon}}\left(\underline{u}^{*}\right) \mathrm{d} \Omega \mathrm{d} t=0, \quad \forall \underline{u}^{*} \in \mathcal{U}_{0}$,

where $\Delta \boldsymbol{\varepsilon}_{i+1}^{R}$ is a known quantity. $\Delta \tilde{\boldsymbol{\varepsilon}}_{i+1}$ can be calculated using the same operator $\mathbb{E}$ as

$\Delta \tilde{\boldsymbol{\varepsilon}}_{i+1}=\mathbb{E} \Delta \boldsymbol{\varepsilon}^{R}$,

and the stress tensor depending on damage is calculated as

$\Delta \tilde{\boldsymbol{\sigma}}_{i+1}=\mathbb{C} \Delta \boldsymbol{\varepsilon}^{R}$.

Finally the total stress and elastic strain tensors in corrective terms are obtained as

$$
\begin{aligned}
\Delta \boldsymbol{\sigma}_{i+1} & =\Delta \boldsymbol{\sigma}_{i+1}^{\prime}+\Delta \tilde{\boldsymbol{\sigma}}_{i+1}, \\
\Delta \boldsymbol{\varepsilon}_{i+1}^{e} & =\Delta \boldsymbol{\varepsilon}_{i+1}^{\prime}+\Delta \tilde{\boldsymbol{\varepsilon}}_{i+1}-\Delta \boldsymbol{\varepsilon}_{i+1}^{p} .
\end{aligned}
$$

\subsection{Hybrid method to construct the PGD reduced-order basis}

For calculating the approximation of the corrective terms in a separable form, it is possible to enrich the reduced-order basis by calculating a new product of a function of space and a function of time, or to re-use the space functions generated at previous iterations and only update the time functions. As updating time functions is less expensive, this strategy is considered at the beginning of the global stage of every LATIN iteration, then enriching the reduced-order basis is done only if necessary as shown on Fig. 2.

\subsubsection{Update of the time functions}

Considering that $m$ pairs have been generated at the end of the global stage of LATIN iteration $i$, at LATIN iteration $i+1$ the objective is to re-use the spatial basis. This phase is thus equivalent as a POD computation on the current PGD basis. The quantities of interest which depend on plastic deformation can be written as

$$
\begin{aligned}
\Delta \dot{\boldsymbol{\varepsilon}}_{i+1}^{p} & =\sum_{j=1}^{m} \Delta \dot{\lambda}_{j}(t) \overline{\boldsymbol{\varepsilon}}_{j}^{p}(\underline{x}), \\
\Delta \boldsymbol{\sigma}_{i+1}^{\prime} & =\sum_{j=1}^{m} \Delta \lambda_{j}(t) \mathbb{C} \overline{\boldsymbol{\varepsilon}}_{j}^{p}(\underline{x}) .
\end{aligned}
$$

These updates of the time functions are calculated from the minimisation of a mechanical residual which is defined by the norm of the search direction operator, i.e.

$$
\left\{\Delta \lambda_{j}\right\}_{j=1}^{m}=\underset{\left\langle\Delta \lambda_{i}\right\}_{j=1}^{m}}{\arg \min }\left\|\sum_{j=1}^{m} \Delta \dot{\lambda}_{j} \overline{\boldsymbol{\varepsilon}}_{j}^{p}-\mathbf{H}_{\sigma} \sum_{j=1}^{m} \Delta \lambda_{j} \mathbb{C}_{\overline{\boldsymbol{\varepsilon}}}^{p}+\overline{\boldsymbol{\Delta}}_{i+1}\right\|_{\mathbf{H}_{\sigma}^{-1}} .
$$

The minimisation problem gives a multi-variable differential equation, which is solved using discontinuous Galerkin method of order zero. More information on discontinuous Galerkin method for solving minimisation problems in LATIN method can be found in [29,42]. Subsequently the total stress and elastic strain are calculated using Eq. (57). After the calculation of all the quantities of interest, if the correction provided by the update of the time functions is not satisfactory, a new space-time pair will be added. For a minimisation problem both in space and in time, a criterion for adding a space-time pair was suggested in [43]. Currently, the criterion to enrich the reduced-order basis is estimated by the saturation of the error indicator. Similar criterion was proposed in [19], a modified version is given as,

$\zeta=\frac{\xi_{i}-\xi_{i+1}}{\xi_{i}+\xi_{i+1}}$.

If this indicator is larger than a particular pre-defined value $\zeta^{\text {tol }}$, the approximation for this LATIN iteration is considered satisfactory. Then, the internal variables are calculated before pursuing with the subsequent LATIN iteration. At the contrary, if the indicator is lower than $\zeta^{\text {tol }}$, a space-time PGD pair is added to enrich the reduced-order basis.

\subsubsection{Addition of space-time modes}

To enrich the reduced-basis, a hybrid strategy is used, that combines a Galerkin formulation to approximate the space fields and a minimisation technique to estimate the corresponding time functions [26]. The search direction equation during the stage of enrichment becomes

$\Delta \dot{\boldsymbol{\varepsilon}}_{i+1}^{p}-\mathbf{H}_{\sigma} \Delta \boldsymbol{\sigma}_{i+1}^{\prime}+\overline{\boldsymbol{\Delta}}_{i+1}=0$,

with

$\overline{\boldsymbol{\Delta}}_{i+1}=\mathbf{H}_{\sigma}\left(\hat{\boldsymbol{\sigma}}_{i+1 / 2}-\boldsymbol{\sigma}_{i+1}^{\text {up }}\right)-\left(\hat{\dot{\boldsymbol{\varepsilon}}}_{i+1 / 2}^{p}-\dot{\boldsymbol{\varepsilon}}_{i+1}^{p, \text { up }}\right)$, 
where $\dot{\boldsymbol{\varepsilon}}_{i+1}^{p \text {, up }}$ and $\boldsymbol{\sigma}_{i+1}^{\text {up }}$ are the quantities obtained from the update stage. The quantities of interest are written in a separable form as

$$
\begin{aligned}
\Delta \dot{\boldsymbol{\varepsilon}}_{i+1}^{p} & =\Delta \dot{\lambda}_{m+1}(t) \overline{\boldsymbol{\varepsilon}}_{m+1}^{p}(\underline{x}), \\
\Delta \boldsymbol{\sigma}_{i+1}^{\prime} & =\Delta \lambda_{m+1}(t) \mathbb{C} \overline{\boldsymbol{\varepsilon}}_{m+1}^{p}(\underline{x}) .
\end{aligned}
$$

A fixed point algorithm is used to calculate the spacetime pair. All the superscripts and subscripts that have been used previously are dropped for simplicity for the following development to calculate the space function by a Galerkin technique. The strain partition relation combined with the state equation is written as

$\Delta \dot{\boldsymbol{\varepsilon}}^{\prime}=\Delta \dot{\boldsymbol{\varepsilon}}^{p}+\mathbf{C}^{-1} \Delta \dot{\boldsymbol{\sigma}}^{\prime}$,

where $\Delta \dot{\boldsymbol{\varepsilon}}^{p}$ is obtained from Eq. (61) and $\Delta \boldsymbol{\sigma}^{\prime}=\lambda \overline{\boldsymbol{\sigma}}$ with $\overline{\boldsymbol{\sigma}}=\lambda \mathbb{C} \overline{\boldsymbol{\varepsilon}}^{p}$. The kinematic admissibility condition in rate form is written as

$\int_{[0, T] \times \Omega} \Delta \dot{\boldsymbol{\varepsilon}}^{\prime}: \boldsymbol{\sigma}^{*} \mathrm{~d} \Omega \mathrm{d} t=0, \quad \forall \boldsymbol{\sigma}^{*} \in \mathcal{S}_{0}$,

which leads, introducing $\langle\cdot\rangle=\int_{[0, T]} \cdot \mathrm{d} t$ and $\sigma^{*}=\lambda \overline{\boldsymbol{\sigma}}$, to

$\int_{\Omega}\left[\left\langle\mathbf{H}_{\sigma} \lambda^{2}\right\rangle \overline{\boldsymbol{\sigma}}+\langle\dot{\lambda} \lambda\rangle \mathbf{C}^{-1} \overline{\boldsymbol{\sigma}}-\langle\overline{\boldsymbol{\Delta}} \lambda\rangle\right]: \overline{\boldsymbol{\sigma}}^{*} \mathrm{~d} \Omega=0, \quad \forall \overline{\boldsymbol{\sigma}}^{*} \in \mathcal{S}_{0}$.

By defining $\overline{\tilde{\varepsilon}}$ such that

$\overline{\tilde{\varepsilon}}=\mathbf{W}^{-1} \overline{\boldsymbol{\sigma}}-\bar{\delta}$,

with $\mathbf{W}^{-1}=\left\langle\mathbf{H}_{\sigma} \lambda^{2}\right\rangle+\langle\dot{\lambda} \lambda\rangle \mathbf{C}^{-1}$ and $\bar{\delta}=\langle\overline{\bar{\Delta}} \lambda\rangle$, it is established that

$\int_{\Omega} \overline{\tilde{\varepsilon}}: \overline{\boldsymbol{\sigma}}^{*} \mathrm{~d} \Omega=0, \quad \forall \overline{\boldsymbol{\sigma}}^{*} \in \mathcal{S}_{0}$

The static admissibility to zero of $\bar{\sigma}$ reads

$\int_{\Omega} \overline{\boldsymbol{\sigma}}: \boldsymbol{\varepsilon}\left(\underline{\bar{u}}^{*}\right) \mathrm{d} \Omega=0, \quad \forall \underline{\bar{u}}^{*} \in \mathcal{U}_{0}$,

which is rewritten using Eq. (67) and by introducing displacement $\overline{\tilde{u}} \in \mathcal{U}_{0}$ such that $\boldsymbol{\varepsilon}(\overline{\tilde{u}})=\overline{\tilde{\varepsilon}}$ :

$$
\int_{\Omega} \mathbf{W} \boldsymbol{\varepsilon}(\underline{\tilde{\tilde{u}}}): \boldsymbol{\varepsilon}\left(\underline{\bar{u}}^{*}\right) \mathrm{d} \Omega=-\int_{\Omega} \mathbf{W} \bar{\delta}: \boldsymbol{\varepsilon}\left(\underline{\bar{u}}^{*}\right) \mathrm{d} \Omega=0, \quad \forall \underline{\bar{u}}^{*} \in \mathcal{U}_{0} .
$$

This problem is solved classically to obtain $\underline{\tilde{\tilde{u}}}$ and thereby calculate the associated strain $\overline{\tilde{\varepsilon}}$. The space function $\overline{\boldsymbol{\varepsilon}}_{m+1}^{p}$ is thereby calculated as,

$\overline{\boldsymbol{\varepsilon}}_{m+1}^{p}=\frac{1}{\langle\lambda \dot{\lambda}\rangle}\left[\overline{\tilde{\boldsymbol{\varepsilon}}}-\langle\lambda \dot{\lambda}\rangle \mathbf{C}^{-1} \mathbf{W}(\overline{\tilde{\boldsymbol{\varepsilon}}}+\overline{\boldsymbol{\delta}})\right]$

Then the time function $\lambda_{m+1}$ is calculated using a minimisation technique similar to the update stage

$\lambda_{m+1}=\underset{\lambda_{m+1}}{\arg \min }\left\|\dot{\lambda}_{m+1} \overline{\boldsymbol{\varepsilon}}_{m+1}^{p}-\mathbf{H}_{\sigma} \lambda_{m+1} \mathbb{C} \overline{\boldsymbol{\varepsilon}}_{m+1}^{p}+\overline{\boldsymbol{\Delta}}_{i+1}\right\|_{\mathbf{H}_{\sigma}^{-1}}$.

The spatial basis is orthonormalised using Gram-Schmidt algorithm [46]. The new space function is orthonormalised with respect to the previously existing spatial bases, and in the numerical process all the former time functions are updated. The new time function is also modified and the corresponding space-time pair may be rejected if the corresponding modified time function has an insignificant norm. Finally, the global stage is concluded by estimating the hardening and damage variables.

\subsection{Internal variables at the global stage}

As the internal variables are local in space, they are calculated without any PGD approximation, but by simply solving first order ordinary differential equations in time locally at each Gauss point (GP). The hardening variables represented by $\boldsymbol{X}$ and $\boldsymbol{Z}$ are obtained by the state laws and search direction operators. The "normal" formulation allows to represent the hardening state laws as

$Z_{i+1}=\Lambda \boldsymbol{X}_{i+1}$

where $\boldsymbol{\Lambda}$ is a linear operator describing the "normal" formulation. The search direction equation for hardening variables (26a) combined with the state equation (73) can be written as

$$
\begin{aligned}
-\left(\dot{\boldsymbol{X}}_{i+1}-\hat{\dot{\boldsymbol{X}}}_{i+1 / 2}\right) & =\mathbf{H}_{Z}\left(\boldsymbol{Z}_{i+1}-\hat{\boldsymbol{Z}}_{i+1 / 2}\right) \\
& =\mathbf{H}_{Z}\left(\boldsymbol{\Lambda} \boldsymbol{X}_{i+1}-\hat{\boldsymbol{Z}}_{i+1 / 2}\right) .
\end{aligned}
$$

For damage, the search direction operator $\mathbf{b}^{-}$is taken to be zero, leading to

$\dot{D}_{i+1}=\hat{\dot{D}}_{i+1 / 2}$

and finally, the energy release rate $Y_{i+1}$ is calculated through Eq. (4d). 
Fig. 3 A bar in traction

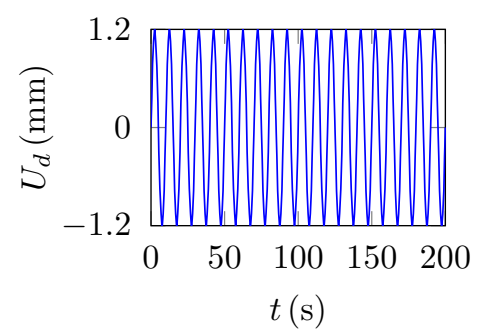

\subsection{Relaxation of the solution field and convergence criterion}

To ensure convergence of the algorithm, the global step is modified by adding a relaxation step. From the solution set $\breve{s}_{i+1}$ obtained at the end of the iterative PGD process, the approximation provided by the global stage is defined as $\mu \breve{\boldsymbol{s}}_{i+1}+(1-\mu) \boldsymbol{s}_{i}$. The relaxation parameter $\mu$ is chosen to be 0.8 . The convergence of the iterative algorithm is determined by a relative LATIN indicator. This indicator is basically the distance between the local solution and the global solution, given by

$\xi=\frac{\left\|\hat{\boldsymbol{s}}_{i+1 / 2}^{p}-\boldsymbol{s}_{i+1}^{p}\right\|}{\left\|\hat{\boldsymbol{s}}_{i+1 / 2}^{p}\right\|+\left\|\boldsymbol{s}_{i+1}^{p}\right\|}$,

with

$$
\begin{aligned}
\left\|\boldsymbol{s}^{p}\right\|^{2}= & \int_{[0, T] \times \Omega}\left(\boldsymbol{\sigma}: \mathbf{H}_{\sigma} \boldsymbol{\sigma}+\boldsymbol{Z}: \mathbf{H}_{Z} \boldsymbol{Z}+\dot{\boldsymbol{\varepsilon}}^{p}: \mathbf{H}_{\sigma}^{-1} \dot{\boldsymbol{\varepsilon}}^{p}+\boldsymbol{\varepsilon}^{e}: \mathbf{C} \boldsymbol{\varepsilon}^{e}\right. \\
& \left.+\dot{\boldsymbol{X}}: \mathbf{H}_{Z}^{-1} \dot{\boldsymbol{X}}\right) \mathrm{d} \Omega \mathrm{d} t
\end{aligned}
$$

It has to be noted that the relaxation parameter $\mu$, the regularisation coefficient $\zeta$, and the error indicator tolerance $\xi$ mostly affect the rate of convergence and not the convergence itself. The values of these parameters that have been used here are from the numerical experiments published in previous works $[25,41]$.

\section{Numerical examples}

The innovative algorithm has been tested on academic examples in one-dimensional and two-dimensional cases.

\subsection{Bar under traction}

The first test problem considered is a bar in traction represented in Fig. 3. The geometry of the structure is defined by the length $L=1000 \mathrm{~mm}$ and area of cross section $A=100 \mathrm{~mm}^{2}$. The bar is constrained at $x=0$. At $x=L$, a sinusoidal prescribed displacement loading of amplitude $1.210^{-3} L$ is applied with a time period $\Delta T=10 \mathrm{~s}$, for 20
Table 1 Material properties

\begin{tabular}{llll}
\hline$E$ & $134,000 \mathrm{MPa}$ & & \\
$\nu$ & 0.3 & & \\
$R_{\infty}$ & $30 \mathrm{MPa}$ & & \\
$\gamma$ & $2 \mathrm{MPa}$ & & \\
$C$ & $5500 \mathrm{MPa}$ & & \\
$a$ & $250 \mathrm{MPa}$ & & \\
$k_{D}$ & $2.778 \mathrm{MPa}^{-n_{d}} \mathrm{~s}^{-1}$ & & \\
$n_{D}$ & 2 & & \\
$K$ & $1220 \mathrm{MPa} \mathrm{s}^{1 / n}$ & & mat. 3 \\
$n$ & 2.5 & & \\
$k$ & $K^{-n}$ & mat. 2 \\
$h$ & 0.2 & $82.5 \mathrm{MPa}$ & \\
$\sigma_{y}$ & mat. 1 & & \\
& $80 \mathrm{MPa}$ & & \\
\hline
\end{tabular}

cycles. The structure is composed of three different elastoviscoplastic materials denoted by mat. 1, mat. 2 and mat. 3 . The materials are distinguished by the yield stress $\sigma_{y}$. The material considered is a $\mathrm{Cr}-\mathrm{Mo}$ steel at $580^{\circ} \mathrm{C}$ and its properties are given in Table 1 [32].

The discretisation in space is done with classical finite element scheme and 90 linear bar elements are used to discretise the structure such that each part has the same number of elements. For the time discretisation, the time step chosen is $0.1 \mathrm{~s}$, so for the given loading there are 100 time elements per cycle and in total 2000 time elements. In the computational model, the criterion $\zeta^{\text {tol }}$ to enrich the reduced-order basis is taken to be 0.1 . The algorithm is stopped if the saturation parameter $\zeta$ is lower than $10^{-4}$. The search direction operators have been defined in [24] and the formulation is extended here to incorporate damage:

$$
\begin{aligned}
\mathbf{H}_{\sigma}= & k n\left\langle f^{p}\right\rangle^{n-1} \frac{1}{(1-D)^{2}}, \\
\mathbf{H}_{\beta}= & k n\left\langle f^{p}\right\rangle^{n-1}\left(-\operatorname{sign}\left(\frac{\sigma}{1-D}-\beta\right)+\frac{a}{C} \beta\right)^{2} \\
& +k\left\langle f^{p}\right\rangle^{n} \frac{a}{C},
\end{aligned}
$$

$\mathbf{H}_{R}=k n\left\langle f^{p}\right\rangle^{n-1} \gamma\left(1-\frac{\bar{R} \gamma^{1 / 2}}{2 R_{\infty}}\right)^{2}+k\left\langle f^{p}\right\rangle^{n} \frac{\gamma}{2 R_{\infty}}$. 
Fig. 4 Evolution of the LATIN indicator with respect to the number of PGD pairs or LATIN iterations for the bar problem

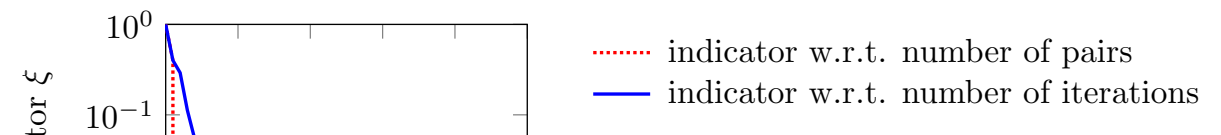

number of pairs $m$ / number of iterations $i$
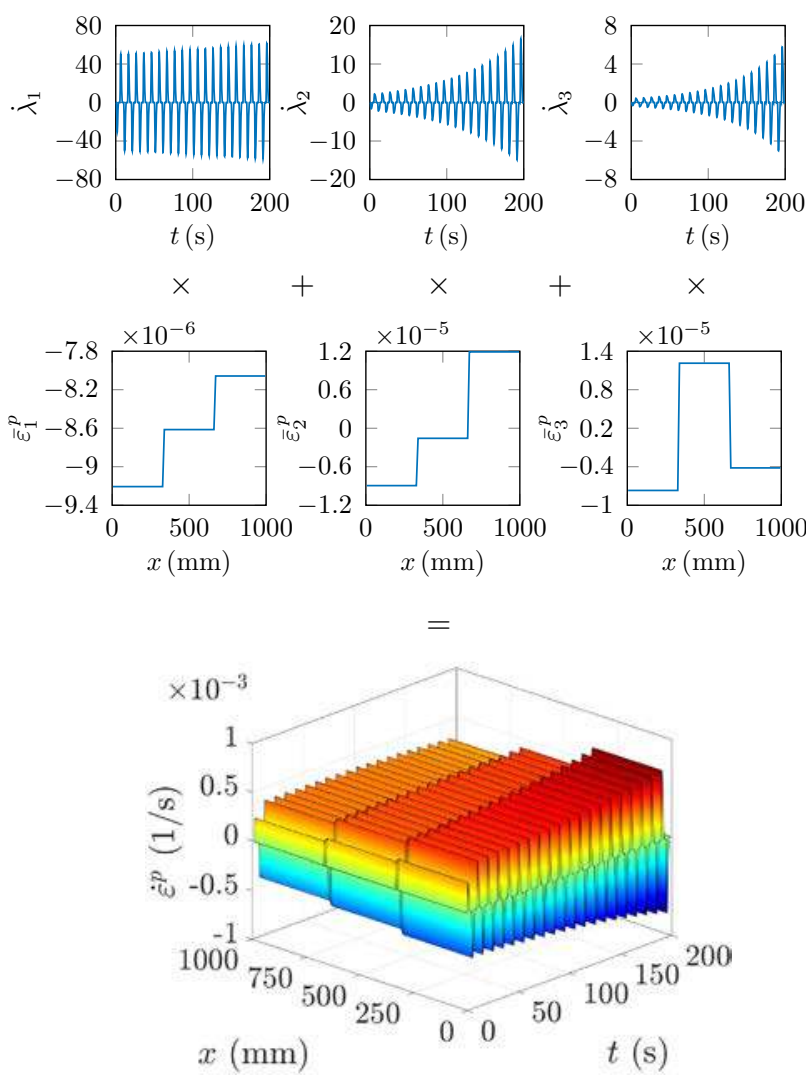

Fig. 5 Space-time modes needed to approximate $\dot{\varepsilon}^{p}(x, t)$ in the bar under cyclic loading at convergence

The convergence of the algorithm is measured by the quantity $\xi$ defined by Eq. (76) and depicted in Fig. 4. The LATIN indicator $\xi$ stagnates at the end which reflects that neither updating the reduced-order basis, nor its enrichment will improve the approximated solution. A finer time step size could decrease the final value of $\xi$. After the convergence of the algorithm, the quantity of interest $\dot{\varepsilon}^{p}$ is obtained as the sum of products of three space-time modes, where the spatial basis is orthonormal (Fig. 5). Due to the rejection of the insignificant space-time modes after orthonormalisation a maximum of three modes is obtained. The time functions generated have increasing amplitude with respect to time.

The quantities of interest (internal variables in rate form and the corresponding associated variables) for the three sections of the bar are depicted in Fig. 6. Due to the particular loading, the stress is constant all over the structure for a particular time, and with respect to time the stress amplitude decreases. The plastic strain rate and kinematic hardening variables have increasing amplitude with respect to time for mat. 1. For mat. 2 and mat. 3, the amplitudes decrease. The isotropic hardening variables show similar behaviour, i.e. for mat. 1 there is a monotonic increase in the amplitude of $\dot{\bar{r}}$, and for mat. 2 and mat. 3, the amplitude of $\dot{\bar{r}}$ decreases. The associated variable $\bar{R}$ increases for all the three materials.

The quantities that indicate loss of stiffness in the material under the direct influence of unilateral condition are depicted in Fig. 7. The damage variable $D$ does not practically increase during the compressive part of the loading and all the evolution takes place during the tensile part. The energy release rate $Y$ during compression is much less than during tension. The elastic strain $\varepsilon^{e}$ also shows a higher value during tension than in compression. Finally after 20 cycles the values of $D$ are $0.22,0.18$ and 0.15 for mat. 1, mat. 2 and mat. 3 respectively.

For general engineering problems both plastic deformation and damage are often highly localised phenomena. The presence of stress raisers (e.g. notches, holes, defects and such others) in most engineering components, concentrates the effect of plasticity and damage to a very limited region, while the rest of the structure remains mostly undamaged and elastic. To depict the localised nature of plasticity and damage, classical two-dimensional mechanical problems are considered in Sects. 5.2 and 5.3.

\section{2 "L" shaped structure}

The test problem considered here is an "L" shaped structure subjected to a concentrated load represented in Fig. 8. The geometry of the structure is defined by the length $L=$ $120 \mathrm{~mm}$, and width $W=20 \mathrm{~mm}$. The structure is filleted 

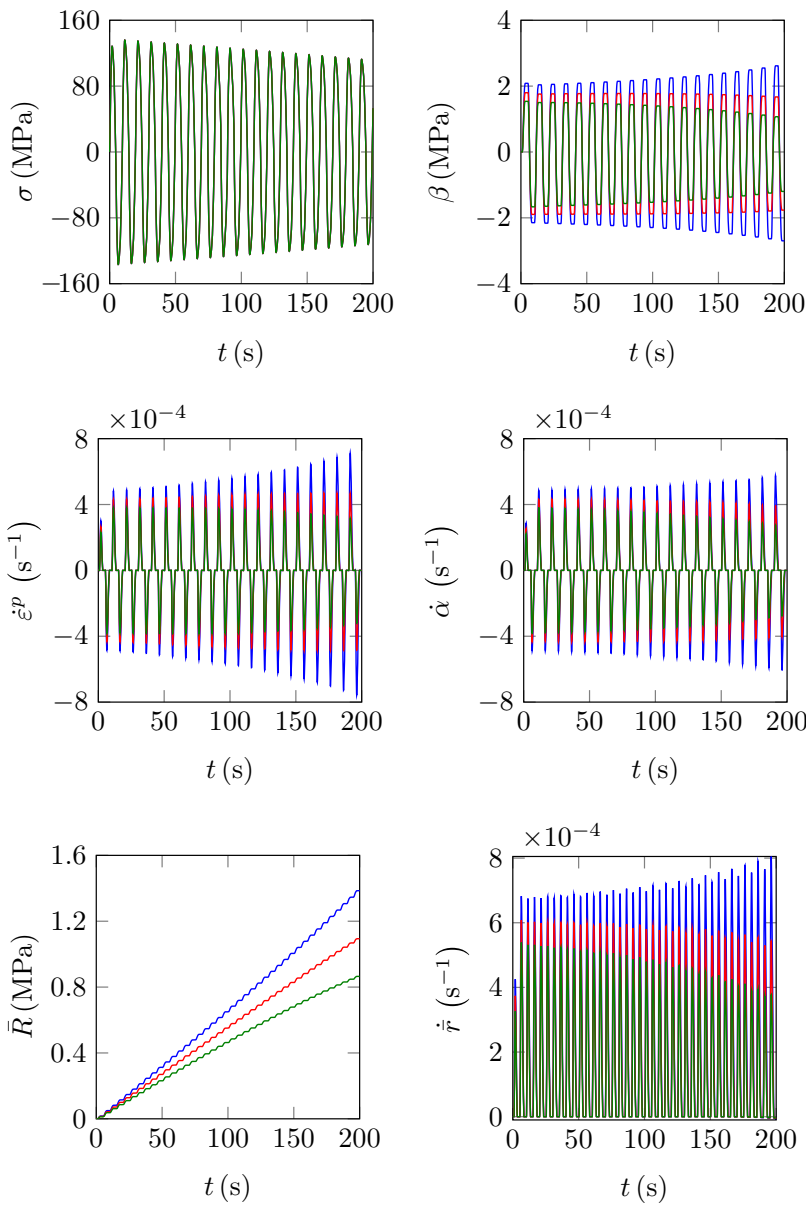

Fig. 6 Evolution of quantities of interest in mat. 1 (blue line), mat. 2 (red line) and mat. 3 (green line) for the bar under cyclic loading. (Color figure online)

at the inside corner with fillet radius $r=5 \mathrm{~mm}$ to avoid stress singularity. The thickness of the structure is taken to be $1 \mathrm{~mm}$. A prescribed sinusoidal displacement $U_{d}(t)$ of amplitude $1.5 \mathrm{~mm}$ is applied with a time period $\Delta T=10 \mathrm{~s}$, for 5 cycles.

The material properties used are the same as in the onedimensional example of Sect. 5.1, and are given in Table 1, with $\sigma_{y}=85 \mathrm{MPa}$. The discretisation in space is done with classical finite element scheme. 277 linear two-dimensional quadrilateral isoparametric plane stress elements with 4 Gauss points per element are used to discretise the structure, which generates 337 nodes. For the time discretisation, the time step chosen is $0.2 \mathrm{~s}$. In the computational model, the criterion to enrich the reduced-order basis $\zeta^{\text {tol }}$ is taken to be $10^{-2}$. The search direction operators $\mathbf{H}_{\sigma}, \mathbf{H}_{\beta}$ and $\mathbf{H}_{R}$ have to be defined for a two-dimensional problem. Theoretically, any symmetric positive definite matrices, used as the search direction operators, suffice to guarantee the convergence of the algorithm, however, the fastest route to convergence is to use the search direction operators associated to the tangent
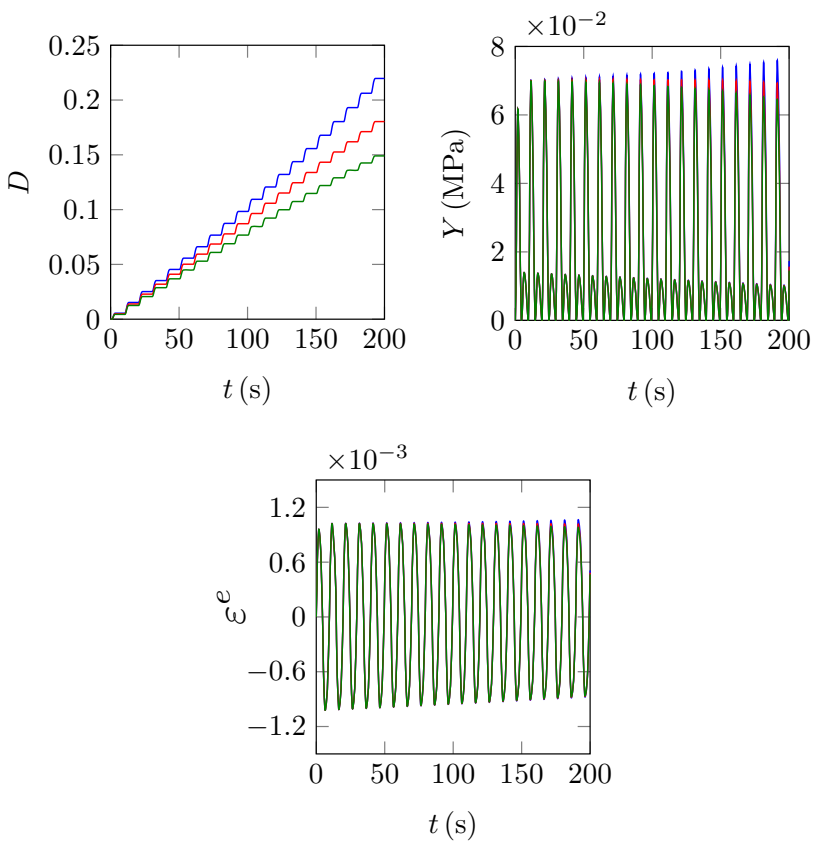

Fig. 7 Evolution of quantities describing damage in mat. 1 (blue line), mat. 2 (red line) and mat. 3 (green line) for the bar under cyclic loading. (Color figure online)

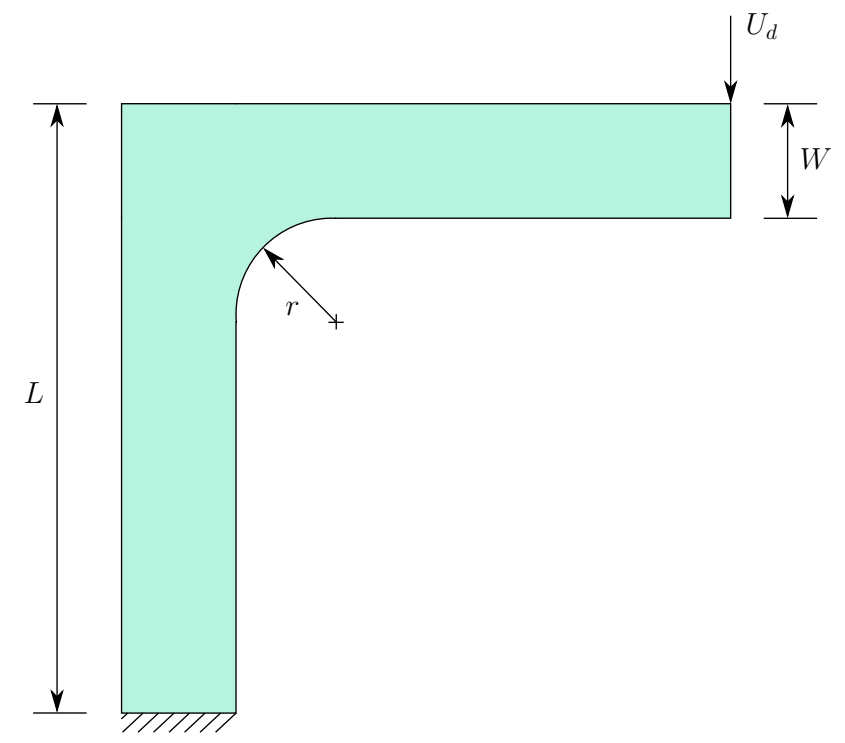

Fig. 8 An "L" shaped structure subjected to a concentrated load

space of the manifold $\boldsymbol{\Gamma}$ [25]. The search direction operator has been specified in [43] for perfect plasticity problems. This idea is extended to include damage and hardening variables,

$\mathbf{H}_{\sigma}=k n\left\langle f^{p}\right\rangle^{n-1} \frac{1}{(1-D)^{2}} \frac{3 / 2 \boldsymbol{\tau}}{J_{2}} \otimes \frac{3 / 2 \boldsymbol{\tau}}{J_{2}}$ 
Fig. 9 Evolution of the LATIN indicator with respect to the number of PGD pairs or LATIN iterations for the "L" shaped structure

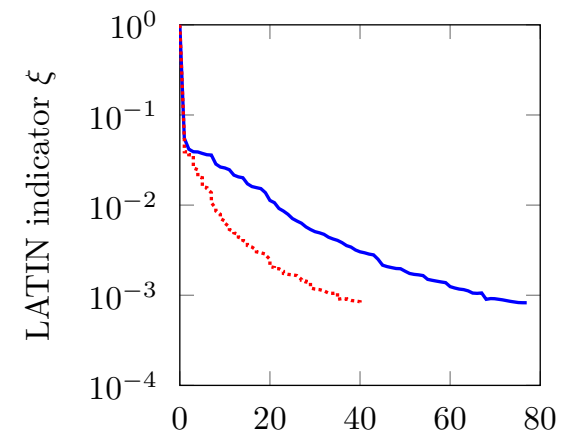

number of pairs $m /$ number of iterations $i$ indicator w.r.t. number of iterations indicator w.r.t. number of pairs
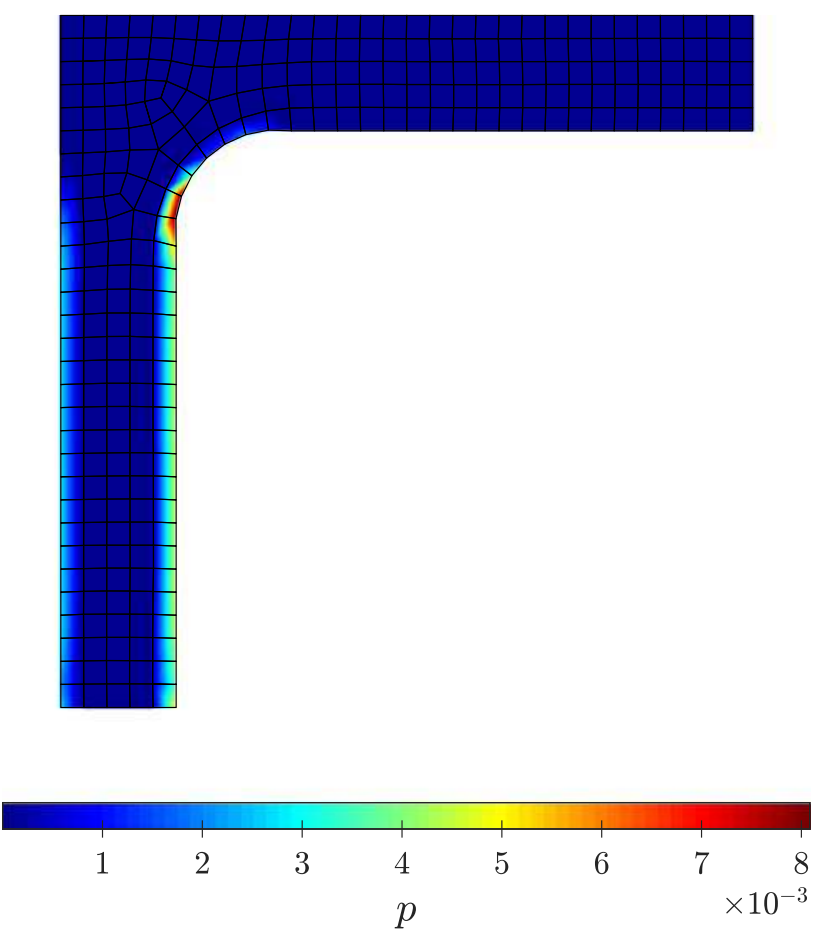

Fig. 10 Distribution of accumulated plastic strain in the "L" shaped structure at $t=T$

$$
\begin{aligned}
& +k\left\langle f^{p}\right\rangle^{n} \frac{1}{(1-D)^{2}} \frac{3 / 2\left(J_{2} \mathbb{I}-3 / 2 \frac{\tau \otimes \boldsymbol{\tau}}{J_{2}}\right)}{J_{2}^{2}} \\
\mathbf{H}_{\beta}= & k n\left\langle f^{p}\right\rangle^{n-1}\left(-\frac{3 / 2 \boldsymbol{\tau}}{J_{2}}+\frac{a}{C} \mathbb{I} \boldsymbol{\beta}\right) \otimes\left(-\frac{3 / 2 \boldsymbol{\tau}}{J_{2}}+\frac{a}{C} \mathbb{I} \boldsymbol{\beta}\right) \\
& +k\left\langle f^{p}\right\rangle^{n} \frac{3 / 2\left(J_{2} \mathbb{I}-3 / 2 \frac{\tau \otimes \tau}{J_{2}}\right)}{J_{2}^{2}} \frac{a}{C} \mathbb{I} \\
\mathbf{H}_{R}= & k n\left\langle f^{p}\right\rangle^{n-1} \gamma\left(1-\frac{\bar{R} \gamma^{1 / 2}}{2 R_{\infty}}\right)^{2}+k\left\langle f^{p}\right\rangle^{n} \frac{\gamma}{2 R_{\infty}},
\end{aligned}
$$

with $J_{2}=\sqrt{\frac{3}{2} \tau \cdot \tau}$. Unlike the one-dimensional problem introduced in Sect. 5.1, the calculation of the search direction operators is considerably expensive, so they are not calcu- lated at every iteration.The Reference Point Method, a hyper reduction technique, has been proposed in [6], and will be introduced in the future. In the current stage, search direction operators are only updated if the difference between two successive global solutions becomes lower than a certain tolerance. This can be measured by the criterion $\zeta$ defined by Eq. (60) with the tolerance being $10^{-2}$. The decrease in the LATIN indicator $\xi$ is shown in Fig. 9. A total of 35 modes are generated to obtain $\xi=9 \times 10^{-4}$. An indicator value of $5 \times 10^{-2}$ is obtained at the end of iteration 1 . After 21 iterations, the LATIN indicator becomes $9 \times 10^{-3}$ with 8 PGD modes. The indicator seems to saturate at $\xi=8 \times 10^{-4}$, and the total number of modes added saturates to 40 . This saturation of LATIN indicator at a relatively high value is due to the fact that a coarse temporal mesh has been used. For a finer temporal mesh this LATIN indicator can be lowered further, as shown in the next numerical example (Sect. 5.3).

The quantity of interest to describe the contribution of the plastic deformation is the accumulated plastic strain given by

$$
p(\underline{x}, t)=\int_{0}^{t}\left(\frac{2}{3} \dot{\boldsymbol{\varepsilon}}^{p}(\underline{x}, \tau): \dot{\boldsymbol{\varepsilon}}^{p}(\underline{x}, \tau)\right)^{1 / 2} \mathrm{~d} \tau, \forall t \in[0, T] .
$$

The distribution of the accumulated plastic strain at time $t=T$ is depicted in Fig. 10. It is distributed on both sides of the vertical web, with the inner side being predominant and the maximum at the filleted corner of the structure. Although the main part of the structure has not been permanently deformed, some plastic deformation can be observed on the inner side of the vertical web, and to a lesser degree, on the outer side of the web.

The distribution of damage variable $D$ at the end of the loading $(t=T)$ is represented in Fig. 11. The distribution is similar to the one of the accumulated plastic strain, i.e. maximum at the filleted corner. The spread of damage in this region of interest after each load cycle is also represented in Fig. 11. The evolution of damage with respect to time for 
Fig. 11 Damage distribution in the " $L$ " shaped structure at $t=T$ and the spread of damage in the region of interest after certain load cycles
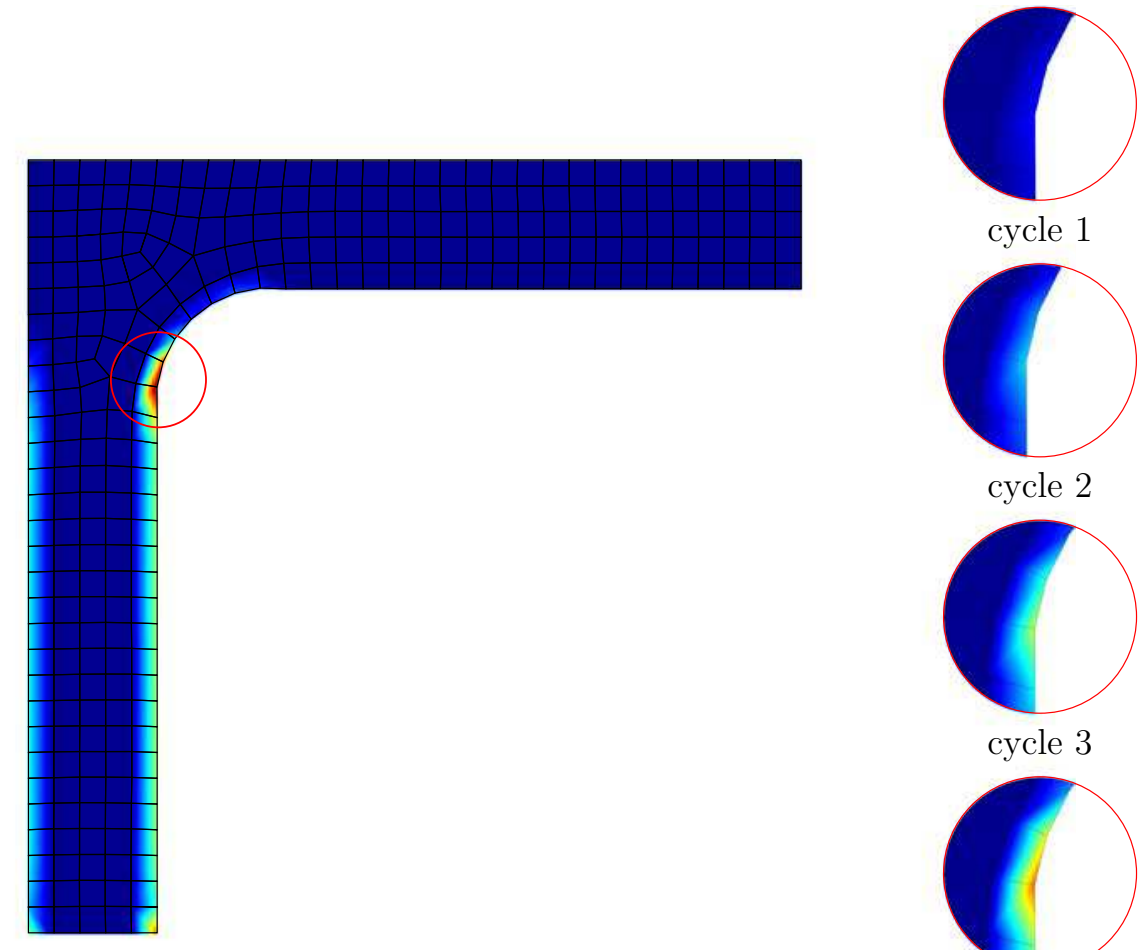

cycle 1

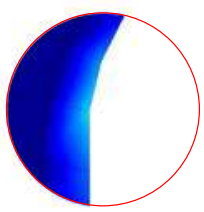

cycle 2

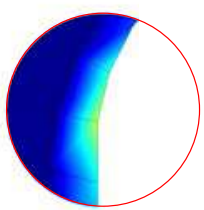

cycle 3

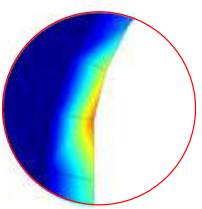

cycle 4

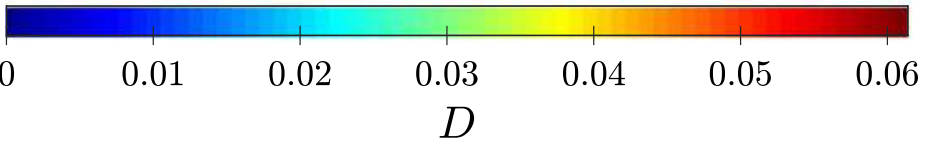

cycle 5

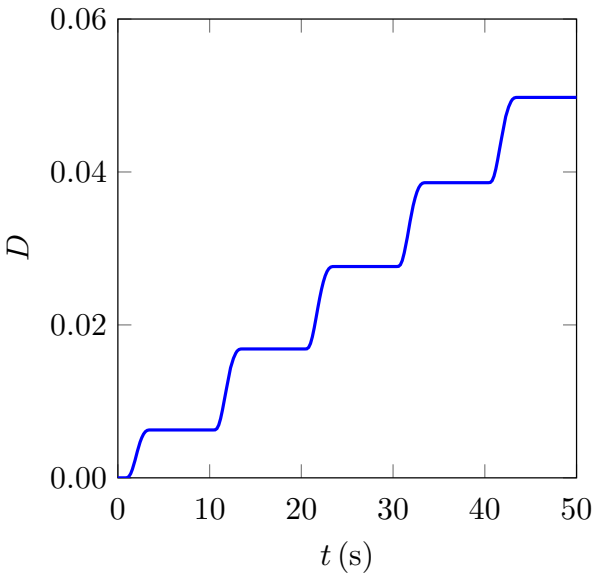

Fig. 12 Damage evolution at the weakest GP of the "L" shaped structure

the weakest GP of this region is shown in Fig. 12 with the maximum value being 0.073 .

The distribution of the residual von Mises stress at the end of the loading is shown in Fig. 13, which similar to the distribution of damage and plastic strain, is concentrated at the filleted corner.

\subsection{Plate with a hole}

The numerical example considered here is a rectangular plate of length $L=40 \mathrm{~mm}$ and width $W=60 \mathrm{~mm}$, with a central circular hole of diameter $\phi=20 \mathrm{~mm}$. The plate is subjected to uniformly distributed sinusoidal displacements of amplitude $0.012 \mathrm{~mm}$ and time period $\Delta T=10 \mathrm{~s}$ on both ends, as shown in Fig. 14, for 10 cycles. Due to symmetry of the structure only a quarter of the plate, with symmetric boundary conditions on the interior of the plate, is considered for the analysis. The thickness of the plate is considered to be $1 \mathrm{~mm}$.

The material properties used are the same as in Table 1 with $\sigma_{y}=85 \mathrm{MPa}$. The discretisation in space is done with classical finite element scheme. 129 linear two-dimensional quadrilateral isoparametric plane stress elements with 4 Gauss points per element are used to discretise the struc- 


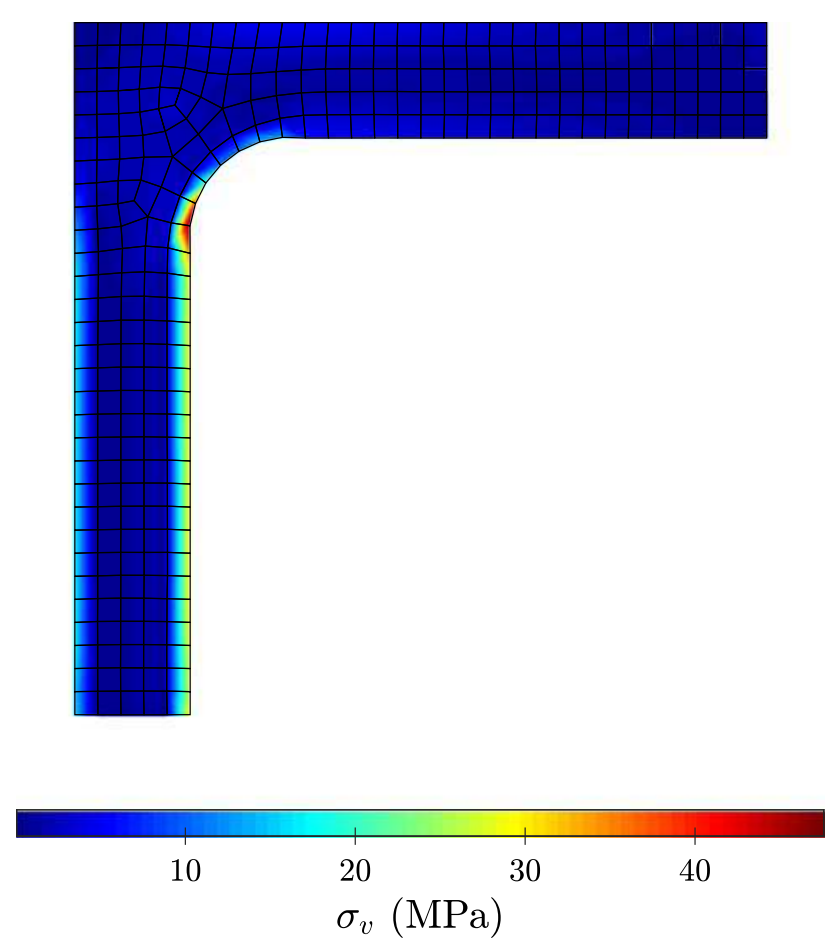

Fig. 13 Distribution of residual von Mises stress in the "L" shaped structure at $t=T$

ture, which generates 154 nodes. For the time discretisation, the time step chosen is $0.1 \mathrm{~s}$.

The primary goal of this example is to depict a comparative study for virgin and pre-damaged structures. The discretised

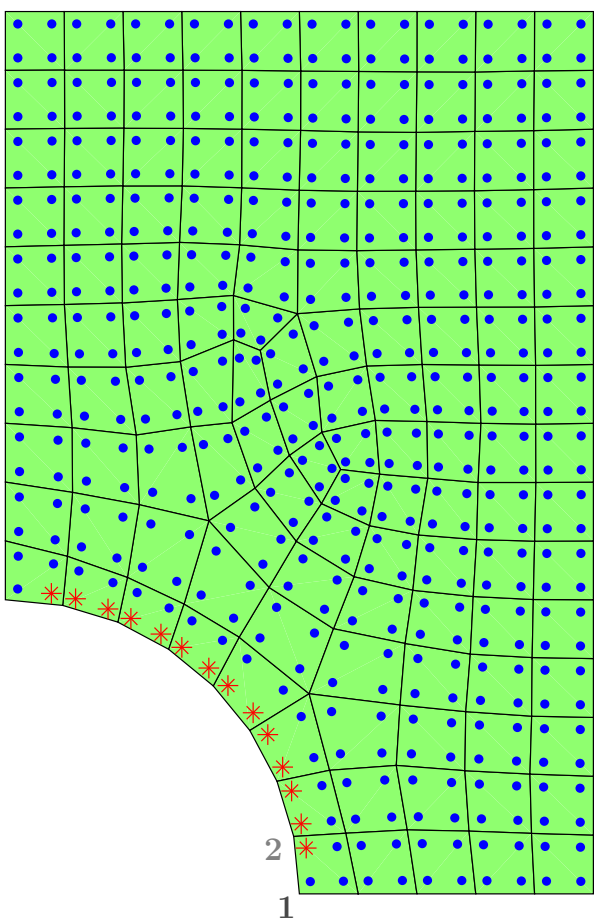

Fig. 15 FE mesh depicting the virgin and pre-damaged Gauss points

structure along with all the GPs is shown in Fig. 15. The first numerical test is to solve the problem considering the structure to be virgin. Thereafter, the GPs near the circumference of the quarter circle except the two corner GPs are assigned
Fig. 14 A plate with a hole subjected to distributed loads and the symmetric part considered for analysis

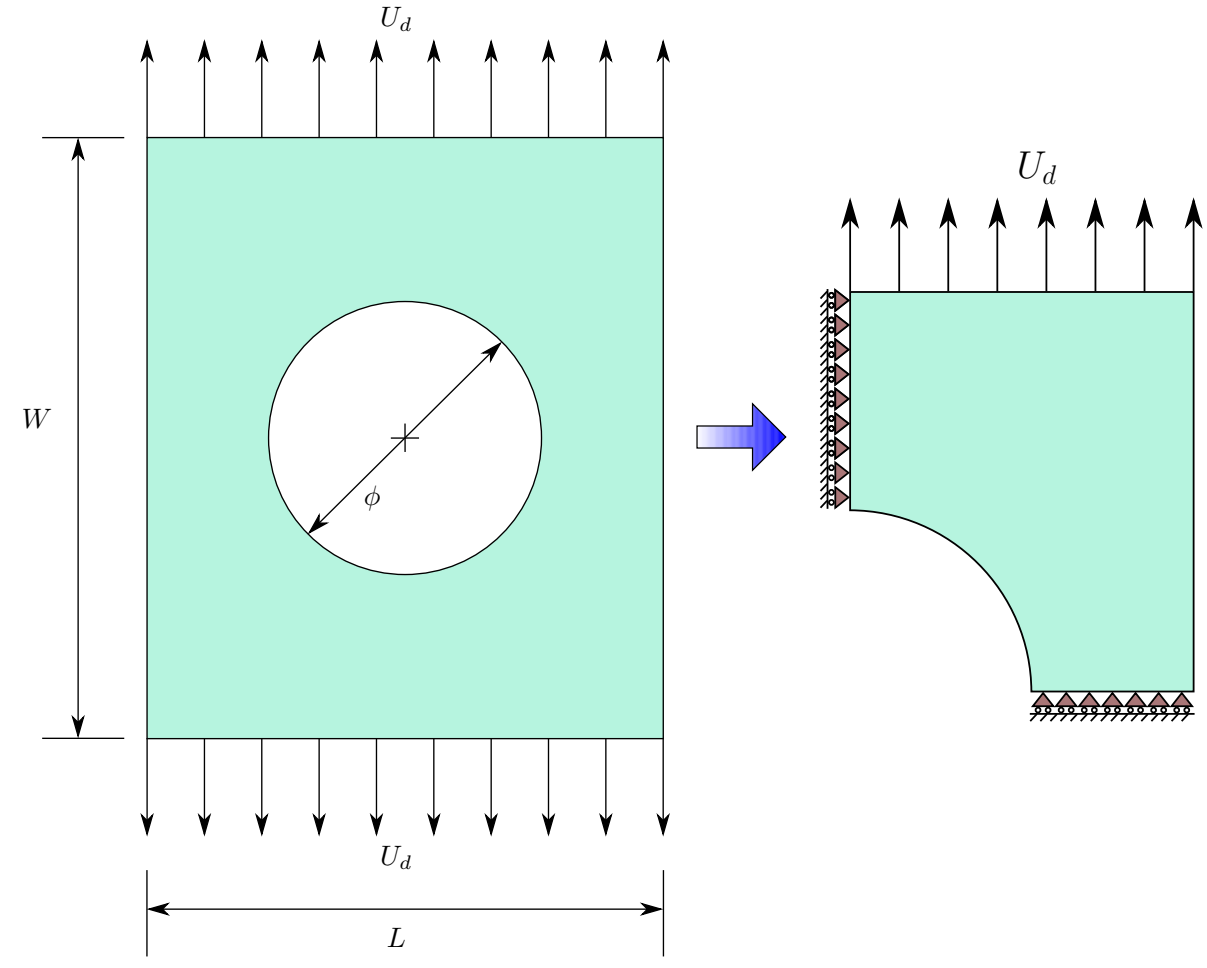


Fig. 16 Evolution of the LATIN indicator with respect to the number of PGD pairs or LATIN iterations for the virgin structure

Fig. 17 Evolution of the LATIN indicator with respect to the number of PGD pairs or LATIN iterations for the pre-damaged structure

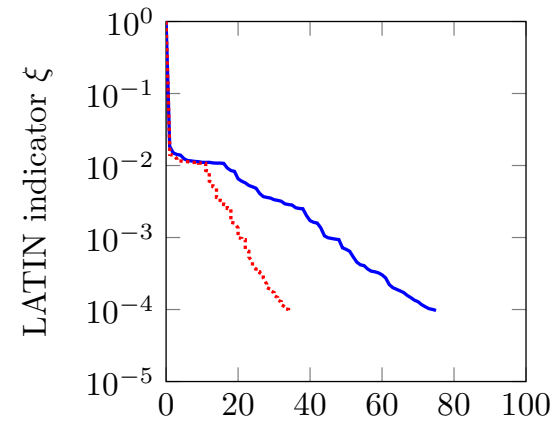

— indicator w.r.t. number of iterations
........ indicator w.r.t. number of pairs

number of pairs $m /$ number of iterations $i$

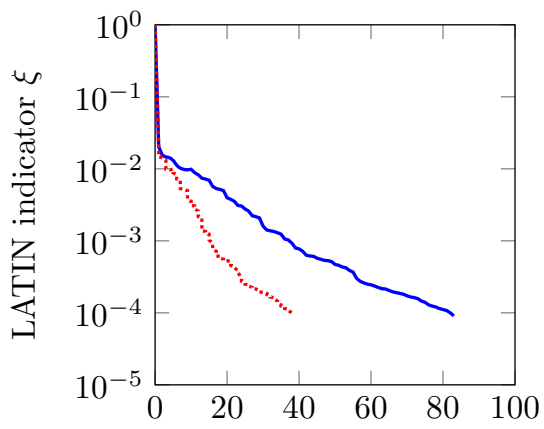

— indicator w.r.t. number of iterations indicator w.r.t. number of pairs

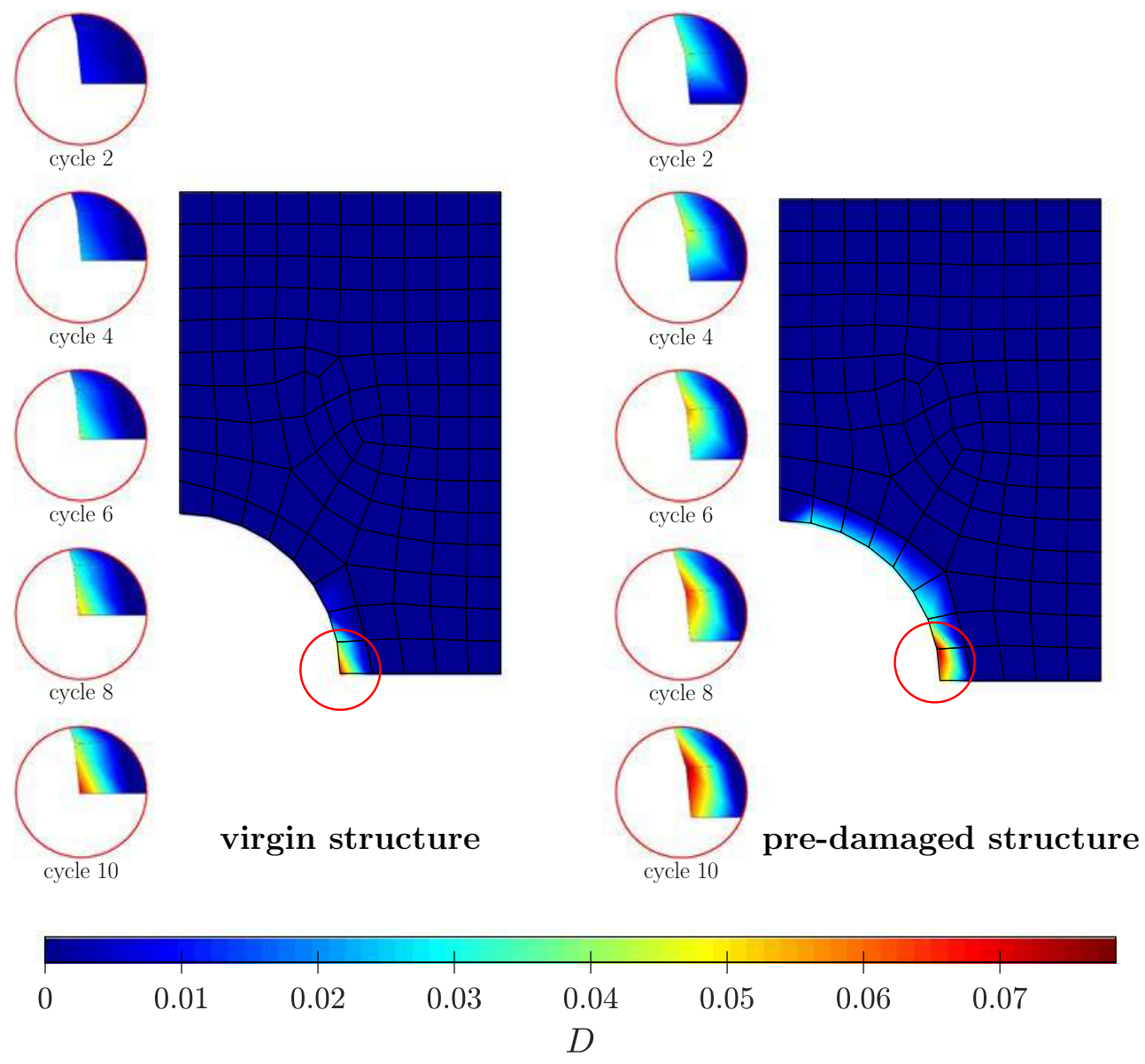

Fig. 18 Comparison of damage distribution at $t=T$ and the spread of damage in the region of interest after certain load cycles 
Fig. 19 Damage evolution at the weakest GP of the plate with hole, i.e. GP 1 for the virgin structure, and GP 2 for the pre-damaged structure

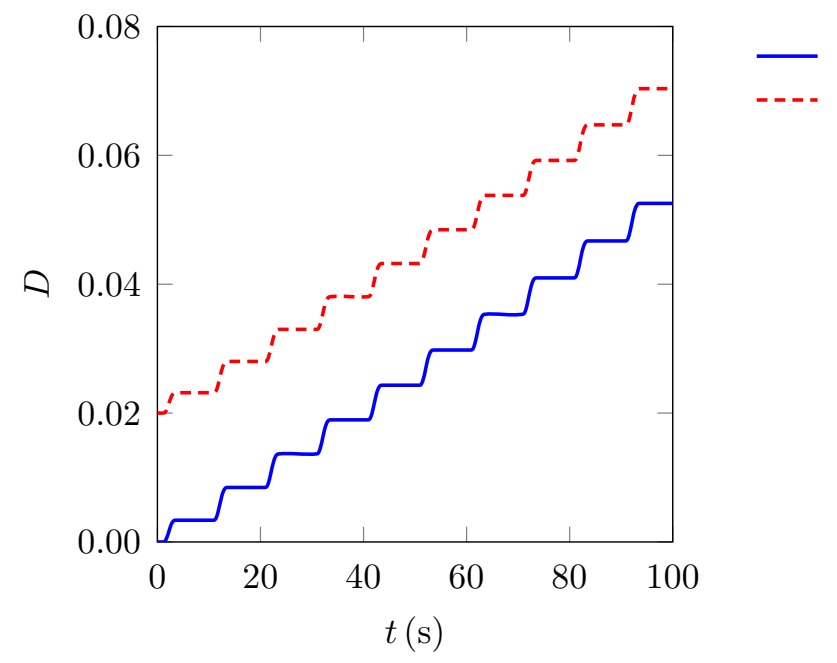

virgin structure pre-damaged structure initial damage values of 0.02 . These pre-damaged GPs are marked with $*$ to distinguish from the virgin GPs which are marked in $\bullet$ (Fig. 15).

The decrease in the LATIN indicator $\xi$ for the virgin structure is shown in Fig. 16. A total of 34 modes are generated to obtain $\xi=9 \times 10^{-5}$. Unlike Sect. 5.2, the LATIN indicator obtained is relatively low. This expected result is due to the usage of finer temporal mesh in this example and it confirms the fact that the LATIN indicator is dependent on temporal discretisation [25] (Fig. 17).

For the pre-damaged structure, compared to the virgin structure, 38 PGD modes are generated to obtain $\xi=$ $9 \times 10^{-5}$. Also a total of 83 iterations are needed, compared to 75 iterations needed for the virgin structure.

The distribution of damage variable $D$, for both the cases, at the end of the loading $(t=T)$ are represented in Fig. 18. The spread of damage for both cases after certain load cycles in the regions of interest are also depicted in Fig. 18. It is quite evident from Fig. 18, that there is a shift of the maximum damaged point in the pre-damaged case, compared to the virgin case. The weakest Gauss point for the virgin structure GP 1 (Fig. 15) is shifted to GP 2 (Fig. 15) for the pre-damaged structure. The evolution of damage for GP 1 and GP 2 for the virgin and pre-damaged cases respectively is shown in Fig. 19, with a maximum of 0.05 for the virgin case and 0.07 for the pre-damaged case.

\section{Conclusion}

In this work, the LATIN-PGD has been developed for solving damage-visco-plasticity problems under cyclic loading. A major difficulty comes from the state laws which can not be transformed into linear relations through an internal variable transformation in order to use the classical LATIN-PGD framework. An extension of the algorithm has been intro- duced for the treatment of this non-linearity and seems to be robust and efficient.

Further works are in progress for the damage detection in the case of fatigue loading involving large number of cycles. For that purpose a new computational framework will be introduced, allowing a multi-scale description of the fields along the time and leading to a strong decrease of the computational cost.

Acknowledgements The authors are grateful to the German Research Foundation (DFG) for funding the research through International Research Training Group 1627.

\section{References}

1. Aguado JV, Huerta A, Chinesta F, Cueto E (2015) Real-time monitoring of thermal processes by reduced-order modeling. Int J Numer Methods Eng 102:991-1017

2. Allier P-E, Chamoin L, Ladevèze P (2015) Proper generalized decomposition computational methods on a benchmark problem: introducing a new strategy based on constitutive relation error minimization. Adv Model Simul Eng Sci 2:1-25

3. Ammar A, Zghal A, Morel F, Chinesta F (2015) On the space-time separated representation of integral linear viscoelastic models. C R Méc 343:247-263

4. Atwell JA, King BB (2001) Proper orthogonal decomposition for reduced basis feedback controllers for parabolic equations. Math Comput Model 33:1-19

5. Bellenger E, Bussy P (1998) Plastic and viscoplastic damage models with numerical treatment for metal forming processes. J Mater Process Technol 80-81:591-596

6. Capaldo M, Guidault P-A, Néron D, Ladevèze P (2017) The reference point method, a hyper reduction technique: application to PGD-based nonlinear model reduction. Comput Methods Appl Mech Eng 322:483-514

7. Carlberg K, Bou-Mosleh C, Farhat C (2010) Efficient nonlinear model reduction via a least-squares petrov-galerkin projection and compressive tensor approximations. Int J Numer Methods Eng 86:155-181

8. Chatterjee A (2000) An introduction to the proper orthogonal decomposition. Curr Sci 78:808-817 
9. Chinesta F, Ladevèze P (eds) (2014) PGD in linear and nonlinear computational solid mechanics. Springer, Vienna, pp 91-152

10. Chinesta F, Ladevèze P, Cueto E (2011) A short review on model order reduction based on proper generalized decomposition. Arch Comput Methods Eng 18(4):395-404

11. Cognard J-Y, Ladevèze $P$ (1993) A large time increment approach for cyclic viscoplasticity. Int J Plast 9:141-157

12. Cojocaru D, Karlsson A (2006) A simple numerical method of cycle jumps for cyclically loaded structures. Int J Fatigue 28:16771689

13. de Souza Neto E, Perić D, Owen D (2008) Computational methods for plasticity. Wiley, Chichester

14. El Halabi F, González D, Sanz-Herrera J, Doblaré M (2016) A PGD-based multiscale formulation for non-linear solid mechanics under small deformations. Comput Methods Appl Mech Eng 305:806-826

15. Galvis J, Kang SK (2014) Spectral multiscale finite element for nonlinear flows in highly heterogeneous media: a reduced basis approach. J Comput Appl Math 260:494-508

16. González D, Alfaro I, Quesada C, Cueto E, Chinesta F (2015) Computational vademecums for the real-time simulation of haptic collision between nonlinear solids. Comput Methods Appl Mech Eng 283:210-223

17. Grepl M, Maday Y, Nguyen N, Patera A (2007) Efficient reducedbasis treatment of nonaffine and nonlinear partial differential equations. Modél Math Anal Numér 41:575-605

18. Guennouni T (1988) Sur une méthode de calcul de structures soumises à des chargements cycliques : l'homogénéisation en temps. Modél Math Anal Numér 22:417-455

19. Heyberger C, Boucard P-A, Néron D (2012) Multiparametric analysis within the proper generalized decomposition framework. Comput Mech 49(3):277-289

20. Kachanov LM (1986) Introduction to continuum damage mechanics, mechanics of elastic stability. Springer, Berlin

21. Kerfriden P, Gosselet P, Adhikari S, Bordas S (2011) Bridging proper orthogonal decomposition methods and augmented Newton-Krylov algorithms: an adpative model reduction for highly nonlinear mechanical problems. Comput Methods Appl Mech Eng 200:850-866

22. Kunish K, Xie L (2005) Pod-based feedback control of the burgers equation by solving the evolutionary hjb equation. Comput Math Appl 49(7-8):5730-5742

23. Ladevèze $P(1989)$ The large time increment method for the analyse of structures with nonlinear constitutive relation described by internal variables. C R Acad Sci Paris 309:1095-1099

24. Ladevèze $P$ (1999) Nonlinear computational structural mechanics, mechanical engineering series. Springer, New York

25. Ladevèze $P$ (1999) Nonlinear computational structural mechanics-new approaches and non-incremental methods of calculation, mechanical engineering series. Springer, New York

26. Ladevèze $P$ (2016) On reduced models in nonlinear solid mechanics. Eur J Mech A Solids 60:227-237

27. Ladevèze P, Nouy A (2003) On a multiscale computational strategy with time and space homogenization for structural mechanics. Comput Methods Appl Mech Eng 192(28):3061-3087

28. Ladevèze P, Néron D, Passieux J-C (2009) On multiscale computational mechanics with time-space homogenization. In: Fish J (ed) Multiscale methods-bridging the scales in science and engineering. Oxford University Press, Oxford, pp 247-282

29. Ladevèze P, Passieux J-C, Néron D (2010) The latin multiscale computational method and the proper generalized decomposition. Comput Methods Appl Mech Eng 199:1287-1296
30. Lemaitre J (1985) Coupled elasto-plasticity and damage constitutive equations. Comput Methods Appl Mech Eng 51:31-49

31. Lemaitre J, Chaboche J-L (1990) Mechanics of solid materials. Cambridge University Press, Cambridge

32. Lemaitre J, Desmorat R (2005) Engineering damage mechanics: ductile, creep, fatigue and brittle failures. Springer, Berlin

33. Lemaitre J, Sermage J, Desmorat R (1999) A two scale damage concept applied to fatigue. Int J Fract 97:67-81

34. Maday Y, Ronquist EM (2004) The reduced-basis element method: application to a thermal fin problem. SIAM J Sci Comput 26(1):240-258

35. Maitournam H, Pommier B, Thomas J-J (2002) Détermination de la réponse asymptotique d'une structure anélastique sous chargement thermodynamique cyclique. C. R. Mec 330:703-708

36. Metoui S, Prulière E, Ammar A, Dau F, Iordanoff I (2014) The proper generalized decomposition for the simulation of delamination using cohesive zone model. Int J Numer Methods Eng 99:1000-1022

37. Murakami S (2012) Continuum damage mechanics: a continuum mechanics approach to the analysis of damage and fracture, solid mechanics and its applications. Springer, Berlin

38. Néron D, Boucard P-A, Relun N (2015) Time-space PGD for the rapid solution of 3D nonlinear parametrized problems in the manyquery context. Int J Numer Methods Eng 103:275-292

39. Nguyen N, Veroy K, Patera A (2005) Certified real-time solution of parametrized partial differential equations, handbook of materials modeling. Springer, Berlin, pp 1523-1558

40. Prud'homme C, Rovas D, Veroyand K, Machiels L, Maday Y, Patera A, Turinici G (2002) Reliable real-time solution of parametrized partial differential equations: reduced-basis output bound methods. J Fluids Eng 124:70-80

41. Relun N, Néron D, Boucard P (2013) A model reduction technique based on the PGD for elastic-viscoplastic computational analysis. Comput Mech 51:83-92

42. Relun N, Néron D, Boucard P-A (2011) Multiscale elasticviscoplastic computational analysis. Eur J Comput Mech 20:379409

43. Relun N, Néron D, Boucard P-A (2013) A model reduction technique based on the PGD for elastic-viscoplastic computational analysis. Comput Mech 51:83-92

44. Rozza G (2005) Reduced-basis methods for elliptic equations in sub-domains with a posteriori error bounds and adaptivity. Appl Numer Math 55(4):403-424

45. Rozza V, Patera AT (2008) Reduced basis approximation and a posteriori error estimation for affinely parametrized elliptic coercive partial differential equations. Arch Comput Methods Eng 15(3):229-275

46. Ruhe A (1983) Numerical aspects of Gram-Schmidt orthogonalization of vectors. Linear Algebra Appl 52:591-601

47. Ryckelynck D, Missoum Benziane D, Cartel S, Besson J (2011) A robust adaptive model reduction method for damage simulations. Comput Mater Sci 50:1597-1605

48. Stolz C (2008) Optimal control approach in nonlinear mechanics. C. R. Mec 336:238-244

49. Veroy K, Patera AT (2005) Certified real-time solution of the parametrized steady incompressible navier-stokes equations: rigorous reduced-basis a posteriori error bounds. Int J Numer Methods Fluids 47:773-788 